\author{
SANDIA REPORT \\ SAND2011-4361 \\ Unlimited Release \\ June 2011
}

\title{
Neutron Counting and Gamma Spectroscopy with PVT Detectors
}

Dean J. Mitchell and Charles Brusseau

Prepared by

Sandia National Laboratories

Albuquerque, New Mexico 87185

Sandia National Laboratories is a multiprogram laboratory managed and operated by Sandia Corporation, a wholly owned Lockheed Martin Corporation, for the United States Department of Energy's

National Nuclear Security Administration under Contract DE-AC04-94AL85000.

Further dissemination only as authorized to U.S. Government agencies and their contractors; other requests shall be approved by the originating facility or higher DOE programmatic authority. 
Issued by Sandia National Laboratories, operated for the United States Department of Energy by Sandia Corporation.

NOTICE: This report was prepared as an account of work sponsored by an agency of the United States Government. Neither the United States Government, nor any agency thereof, nor any of their employees, nor any of their contractors, subcontractors, or their employees, make any warranty, express or implied, or assume any legal liability or responsibility for the accuracy, completeness, or usefulness of any information, apparatus, product, or process disclosed, or represent that its use would not infringe privately owned rights. Reference herein to any specific commercial product, process, or service by trade name, trademark, manufacturer, or otherwise, does not necessarily constitute or imply its endorsement, recommendation, or favoring by the United States Government, any agency thereof, or any of their contractors or subcontractors. The views and opinions expressed herein do not necessarily state or reflect those of the United States Government, any agency thereof, or any of their contractors.

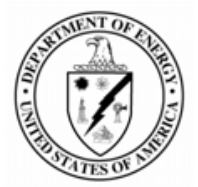




\title{
Neutron Counting and Gamma Spectroscopy with PVT Detectors
}

\author{
Dean J. Mitchell and Charles Brusseau \\ Contraband Detection Technology \\ Sandia National Laboratories \\ P.O. Box 5800 \\ Albuquerque, New Mexico 87185-0782
}

\begin{abstract}
Radiation portals normally incorporate a dedicated neutron counter and a gamma-ray detector with at least some spectroscopic capability. This paper describes the design and presents characterization data for a detection system called PVT-NG, which uses large polyvinyl toluene (PVT) detectors to monitor both types of radiation. The detector material is surrounded by polyvinyl chloride (PVC), which emits highenergy gamma rays following neutron capture reactions. Assessments based on high-energy gamma rays are well suited for the detection of neutron sources, particularly in border security applications, because few isotopes in the normal stream of commerce have significant gamma ray yields above $3 \mathrm{MeV}$. Therefore, an increased count rate for high-energy gamma rays is a strong indicator for the presence of a neutron source. The sensitivity of the PVT-NG sensor to bare ${ }^{252} \mathrm{Cf}$ is 1.9 counts per second per nanogram (cps/ng) and the sensitivity for ${ }^{252} \mathrm{Cf}$ surrounded by $2.5 \mathrm{~cm}$ of polyethylene is $2.3 \mathrm{cps} / \mathrm{ng}$. The PVT-NG sensor is a proof-of-principal sensor that was not fully optimized. The neutron detector sensitivity could be improved, for instance, by using additional moderator.

The PVT-NG detectors and associated electronics are designed to provide improved resolution, gain stability, and performance at high-count rates relative to PVT detectors in typical radiation portals. As well as addressing the needs for neutron detection, these characteristics are also desirable for analysis of the gamma-ray spectra. Accurate isotope identification results were obtained despite the common impression that the absence of photopeaks makes data collected by PVT detectors unsuitable for spectroscopic analysis. The PVT detectors in the PVT-NG unit are used for both gamma-ray and neutron detection, so the sensitive volume exceeds the volume of the detection elements in portals that use dedicated components to detect each type of radiation.
\end{abstract}

\section{Acknowledgement}

The authors gratefully acknowledge support provided by the Department of Homeland Security (DHS) Domestic Nuclear Detection Office (DNDO), under contract DE-AC04-94AL85000. The voxelSPEC development was supported in part by the U.S. Defense Threat Reduction Agency (DTRA), under contract HDTRA1-09-C-0012. 


\section{Contents}

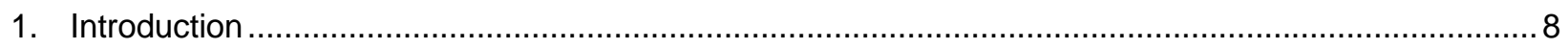

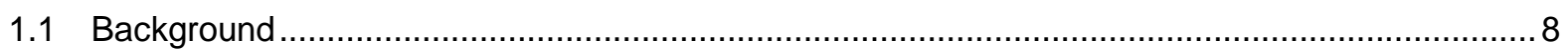

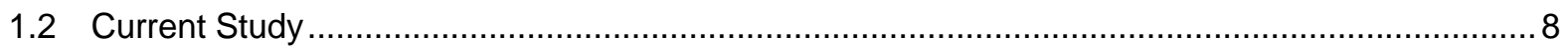

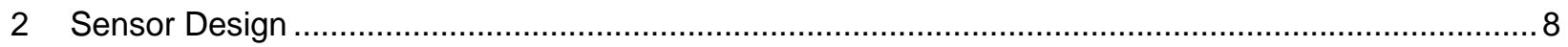

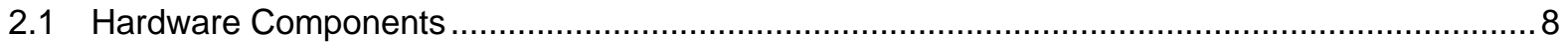

2.2 Electrical Components and Data Acquisition Software ....................................................... 10

2.3 Energy Calibration and Resolution Characterization ............................................................ 10

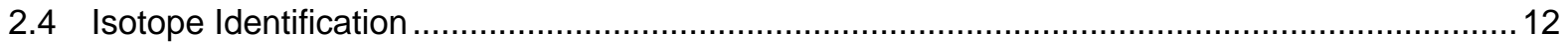

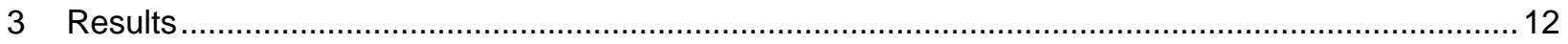

3.1 Detector Response for Gamma-Ray Sources ................................................................ 12

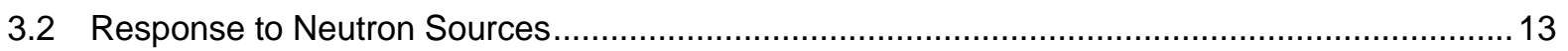

3.2.1 Sensitivity Evaluation Based on Gross Count Rates ................................................. 15

3.2.2 Sensitivity Evaluation Based on Spectroscopic Analysis.......................................... 16

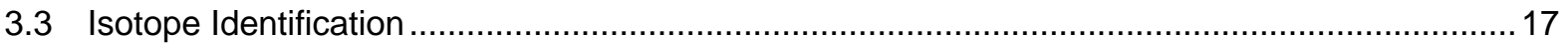

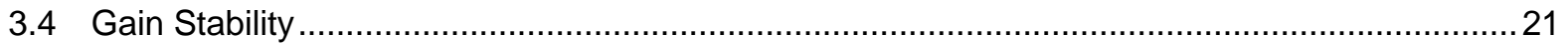

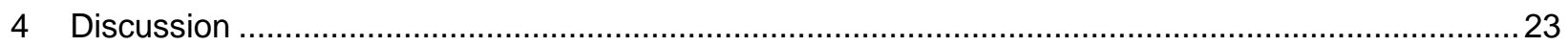

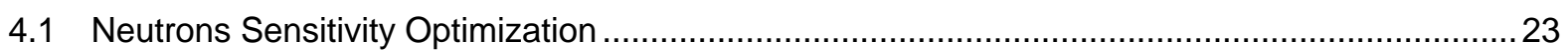

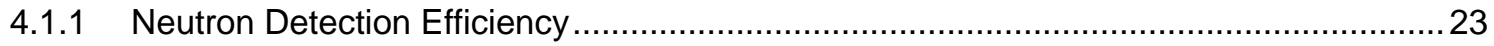

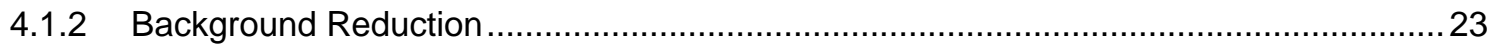

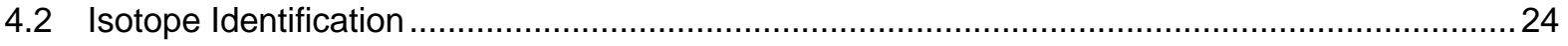

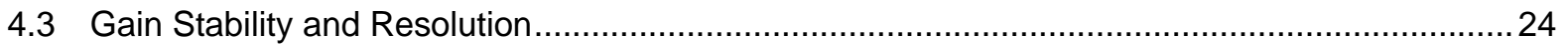

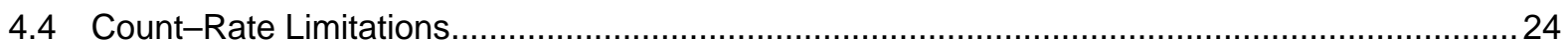

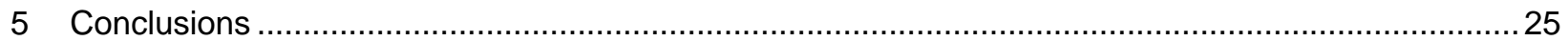

\section{Figures}

Fig. 1. Cross-sectional view of the PVT-NG detector. The front of the detector is at the top of this drawing.

Fig. 2. Front view of the PVT-NG detector.

Fig. 3. A background spectrum measured by MCA1 (black) is compared with the fit of the spectrum (red) that was obtained during the energy-calibration procedure.

Fig. 4. A background spectrum for the coincident-sum spectrum L1 (black) is compared with the fit of the spectrum (red) that was obtained during the energy-calibration procedure.

Fig. 5. Comparison of measured background-subtracted spectra (black) with computed spectra (red) for four calibration sources at a distance of 2 meters.

Fig. 6. Comparison of measured spectra (black) with components associated with the computed gamma-ray leakage from the source (red) and the measured background spectrum (green). The detector was exposed to the radiation sources at a distance of 2 meters. 
Fig. 7. Estimated neutron detection efficiencies as a function of energy for the PVT-NG sensor. The efficiency represents the percent of neutrons that produce a count relative to the total number of neutrons that strike the face.

Fig. 8. The red curve shows the fit to the high-energy portion of a spectrum that was measured while a ${ }^{252} \mathrm{Cf}$ source was present and the black error bars represent the stripped spectrum with \pm 1 -sigma standard deviations

Fig. 9. Comparison of the measured 60-second spectrum for ${ }^{133} \mathrm{Ba}+{ }^{137} \mathrm{Cs}+{ }^{60} \mathrm{Co}^{232} \mathrm{U}$ with components of the spectrum derived from analysis with DHSIsotopeID.

Fig. 10. Comparison of the measured 4-second spectrum for ${ }^{133} \mathrm{Ba}+{ }^{137} \mathrm{Cs}$ with components of the spectrum derived from analysis with DHSIsotopeID. 20

Fig. 11. Comparison of the measured 4-second spectrum for ${ }^{133} \mathrm{Ba}+{ }^{137} \mathrm{Cs}+{ }^{60} \mathrm{Co}^{232} \mathrm{U}$ with components of the spectrum derived from analysis with DHSIsotopeID

Fig. 12. Full-scale energy for logical detectors L1 and L2 with the LED-gain stabilizer turned OFF and the set-point for the piezoelectric coolers at $27 \mathrm{C}$. The measurement started at $4 \mathrm{PM}$ when the outside temperature was $33 \mathrm{C}$ and terminated at $7 \mathrm{AM}$ the following morning, when the outside temperature was $16 \mathrm{C}$. 22

Fig. 13. Full-scale energy for logical detectors L1 and L2 with the LED-gain stabilizer turned ON and the set-point for the piezoelectric coolers at $16 \mathrm{C}$. The measurement started at $9 \mathrm{AM}$ and terminated at $7 \mathrm{AM}$ the following morning. The high for the outside temperature was $33 \mathrm{C}$ and the low was $16 \mathrm{C}$ during this 22-hour measurement sequence.

\section{Tables}

Table 1. Cross-sectional area and background neutron count rate for the PVT-NG sensor........Error! Bookmark not defined.15

Table 2. Minimum detectable activities ( $\mu \mathrm{Ci}$ ) of 252Cf in various configurations.Error! Bookmark not defined.16

Table 3. Summary of analysis results for measurements for gamma-ray emitting sources. 19 


\section{Acronyms}

$\begin{array}{ll}\text { ASP } & \text { advanced spectroscopic portals } \\ \text { BPE } & \text { borated polyethylene } \\ \text { cps/ng } & \text { counts per second per nanogram } \\ \text { FSE } & \text { full-scale energies } \\ \text { GADRAS } & \text { Gamma Detector Response and Analysis Software } \\ \text { HPGe } & \text { high-purity germanium } \\ \text { LED } & \text { light-emitting diodes } \\ \text { FLIR } & \text { FLIR Systems Inc. } \\ \text { MCA } & \text { multichannel analyzers } \\ \text { Nal } & \text { sodium iodine } \\ \text { NEMA } & \text { National Electronics Manufacturers Association } \\ \text { NORM } & \text { naturally-occurring radioactive materials } \\ \text { NS } & \text { nanosecond } \\ \text { PMT } & \text { photomultiplier } \\ \text { PVT } & \text { polyvinyl toluene } \\ \text { PVT-NG } & \text { polyvinyl toluene-neutron gamma } \\ \text { PVC } & \text { polyvinyl chloride } \\ \text { TEAMS } & \text { Technical Evaluation Assessment Monitor Site } \\ \text { VOxelSPEC } & \text { Multichannel analyzer developed by FLIR } \\ \end{array}$


This page intentionally left blank 


\section{Introduction}

\subsection{Background}

Neutrons are normally measured with detectors such as moderated ${ }^{3} \mathrm{He}$ tubes, which are insensitive to gamma-rays by design. An alternative approach for neutron detection is to use features in gamma-ray spectra that are associated with neutron-capture reactions and fission gamma rays. Assessments based on detection of high-energy gamma rays are well suited for this purpose, particularly in border security applications, because few isotopes in the normal stream of commerce have significant gamma ray yields above $3 \mathrm{MeV}$. Therefore, an increased count rate for high-energy gamma rays is a strong indicator for the presence of a neutron source.

A previous investigation demonstrated that high-energy gamma rays recorded by sodium iodide ( $\mathrm{Nal}$ ) detectors of the type used in Advanced Spectroscopic Portals (ASP) are suitable for neutron detection. ${ }^{2}$

\subsection{Current Study}

This report describes the use of large polyvinyl toluene (PVT) (sensors that are normally used in all portals) for combined neutron and gamma detection capability (PVT-NG). The PVT detector is surrounded by polyvinyl chloride (PVC), which produces high-energy gamma rays when neutrons are captured by chlorine. Fission gamma rays and neutron capture by iron in vehicles and cargo also contribute to the detection of neutron sources. Although PVT detectors have negligible photopeak efficiency and generally exhibit poor resolution, they are attractive for portal applications because of their low cost and availability in large sizes. The enhancements reported here in the performance of PVT detectors that allow effective neutron detection also improve the quality of gamma-ray spectra relative to typical portals. Thus, the PVT-NG detector provides clear advantages for both neutron and gamma-ray detection.

Specific advantages of the PVT-NG design are: improved resolution, increased gain stability, and better performance at high-count rates. The improved detection efficiency results because the detector elements in the PVT-NG are twice as thick as PVT detectors in typical portals. While these characteristics are desirable for the application of energy windowing techniques, ${ }^{2}$ this report also explores isotopic identification capabilities using full-spectrum analysis techniques, which do not rely on the identification of photopeaks. ${ }^{3}$ Preliminary results for isotope identification based on spectra recorded by the PVT detector are encouraging.

\section{Sensor Design}

\subsection{Hardware Components}

The PVT-NG sensor is mounted inside a National Electronics Manufacturers Association (NEMA) enclosure $(22 \mathrm{~cm}$ deep $\times 79 \mathrm{~cm}$ wide $\times 222 \mathrm{~cm}$ tall). The panel contains two identical PVT detectors, each of which is $11.1-\mathrm{cm}$ thick, $22.5-\mathrm{cm}$ wide, and $144.4-\mathrm{cm}$ tall. Two detectors are used rather than one larger detector because smaller detectors have better resolution and lower count rates, which improves performance when gamma-ray sources are present. The PVT detectors are surrounded on three sides by $2.54 \mathrm{~cm}$ of PVC, which produces high-energy gamma rays following neutron absorption by chlorine. PVC has several desirable characteristics, including:

- low cost,

- high capture cross section for neutron absorption by chlorine,

- good yield for high-energy gamma rays, and

- the incorporation of hydrogen with the chlorine serves as an internal moderator.

Figure 1 presents a cross-sectional view that identifies the PVT detectors and the surrounding sheets of PVC. A 0.64-cm-thick layer of lead, which surrounds the entire assembly, is comparable to shielding that is used in commercial radiation portals. The detectors are supported by foam cushions and the detector 
assembly is shock-mounted so that the unit can withstand the vibration that it is expected during shipment and installation.

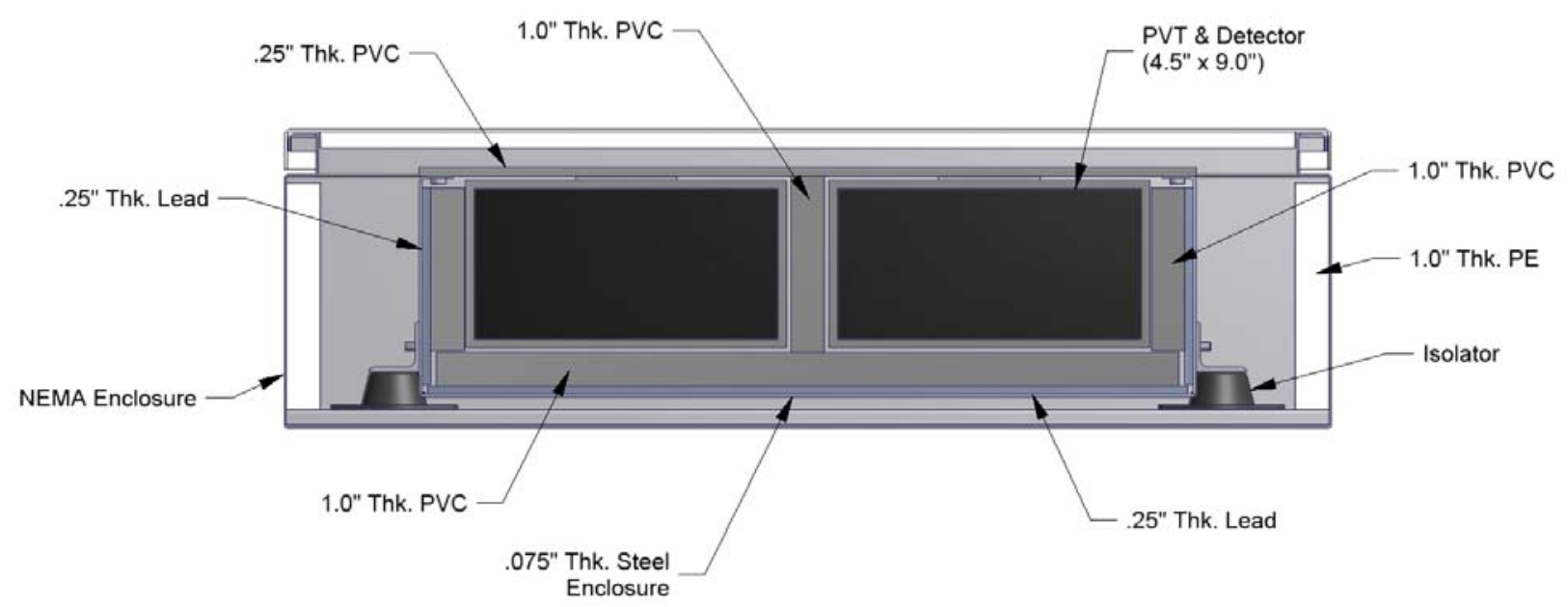

Fig. 1. Cross-sectional view of the PVT-NG detector. The front of the detector is at the top of this drawing.

Figure 2 shows the assembled PVT-NG unit, which is mounted inside a standard portal frame (yellow). The silver units on the top and bottom of the white NEMA enclosure are piezoelectric coolers. Testing at FLIR demonstrated that the voxeISPECs functioned properly at $60^{\circ} \mathrm{C}$, but thermal testing was not performed for the assembled unit. The piezoelectric coolers are probably not required, but they were added to ensure that the unit would function properly during testing in Nevada that is planned for the summer of 2011.

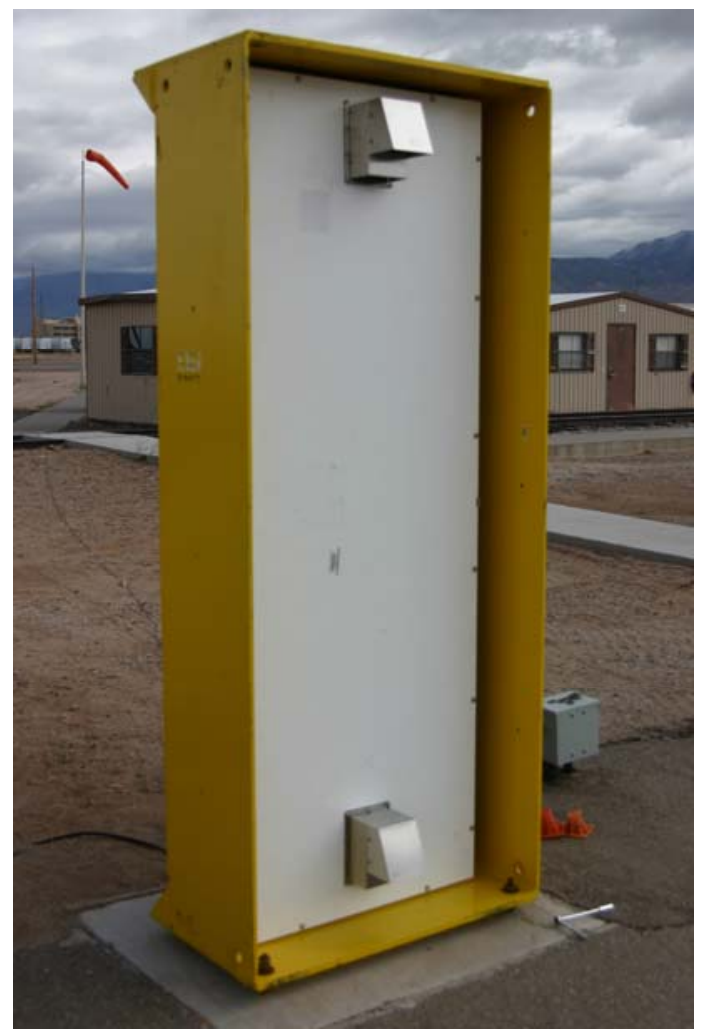

Fig. 2. Front view of the PVT-NG detector. 


\subsection{Electrical Components and Data Acquisition Software}

Each of the two identical detectors has 3-inch diameter photomultiplier (PMT) tubes affixed to the top and bottom of the PVT scintillator. The performance goals were addressed by using recently developed voxeISPEC multichannel analyzers (MCA), ${ }^{4}$ which meet the following requirements:

- Good energy resolution over the range $40 \mathrm{keV}$ to $10 \mathrm{MeV}$

- Operation at high count rates without significant pileup

- Gain stabilization

Preliminary tests indicated that the output from the PVT detectors is relatively insensitive to temperature, but the PMTs exhibit a notable temperature coefficient. FLIR addressed the gain-stabilization requirement by attaching light-emitting diodes (LED) to each of the photomultiplier tubes. The voxeISPECs automatically perform fine gain adjustments to maintain constant amplitude for LED pulses. Spectra are collected in 4096 energy groups over the range 0 to $10 \mathrm{MeV}$. The recorded spectra provide a sufficient number of channels to evaluate isotope identification capabilities for sources that emit gammaray energies below $3 \mathrm{MeV}$ while also extending to encompass high-energy gamma rays produced by neutron-capture reactions.

Spectra are recorded in list mode, which provided sufficient flexibility to enable changes in the data processing during the development phase. Each pulse that is recorded by a voxeISPEC is passed to the data processing computer by Ethernet protocol. EventBuilder software that was provided by FLIR synthesizes spectra from these data. A spectrum acquisition application, which obtains the spectra from EventBuilder, records six spectra for each measurement or measurement interval. Four of the spectra correspond to data collected by the individual MCAs. Calibration parameters, which are established for each MCA, are used to convert pulse areas to equivalent energies. The data are also used to synthesize spectra for two logical detectors, which are referred to as L1 and L2. The logical detectors represent coincident-sum spectra, which record the average of the energies that are recorded by the two MCAs attached to each detector. Pulses are only included in the synthesized spectra when detection events occur in both MCAs within a 100 nanosecond (ns) time window, which reduces low-energy electronic noise. The coincidence-sum spectra exhibit higher-resolution than spectra acquired from individual MCAs because: a) the average of the response of two MCAs on opposite ends of the PVC detector is less sensitive to the location in which the radiation interacts than the response of an individual MCA; and 2) the larger (on average doubled) amount of light collected with the two PMT reduces the statistical fluctuation of the detector signal by a factor of about $\sqrt{2}(\sim 1.4)$. Details associated with the coincidencesum spectra are discussed in more depth in Appendix $A$.

\subsection{Energy Calibration and Resolution Characterization}

A backscatter gating technique, which was developed by Roemer, et al. and by Pausch, et al., enables accurate characterization of the energy calibration and resolution of detectors, such as PVT, which are difficult to characterize because they do not exhibit photopeaks. ${ }^{5,6}$ This technique uses a secondary detector such as Nal or HPGe to measure the energy of scattered photons. While this method provides accurate characterization information, it requires the use of an auxiliary detector and a calibration source. The method that we developed for use with the PVT-NG avoids this complexity by characterizing both the gain and resolution based only on features in the background spectrum. Characterization is performed by fitting the background spectrum in the energy range 1 to $5 \mathrm{MeV}$ with computed spectra for the naturallyoccurring radioactive materials (NORM) plus cosmic radiation. Computed spectra are based on the response function in the Gamma Detector Response Analysis Software (GADRAS) ${ }^{3}$ and radiation leakage from the NORM isotopes in soil . ${ }^{7}$ The variable parameters in this curve-fitting method are the intensities for the component spectra, the full-scale energy, and the resolution at $661 \mathrm{keV}$. The energy offset is assumed to be zero ${ }^{a}$ and skew parameters are asserted based on characterization data (see Section 3.2).

${ }^{\mathrm{a}}$ The voxeISPEC digital signal processing eliminates electronic offset by correcting individual pulses for the actual baseline. 
Although background spectra recorded by PVT detectors don't have distinctive features upon visual inspection, useful characterization information can be obtained. Figure 3 shows the fit that is obtained by analyzing a background spectrum recorded by one of the individual MCAs (MCA1). The full-scale energy is determined to an accuracy of about $2 \%$ for individual detectors (i.e., $200 \mathrm{keV}$ with a full-scale range of $10 \mathrm{MeV}$ ). The 1-sigma uncertainty for the estimated gain is about $50 \mathrm{keV}$ when the coincident-sum spectra are processed by the automated calibration procedure because features are more distinctive due to the improved resolution (see Fig. 4).

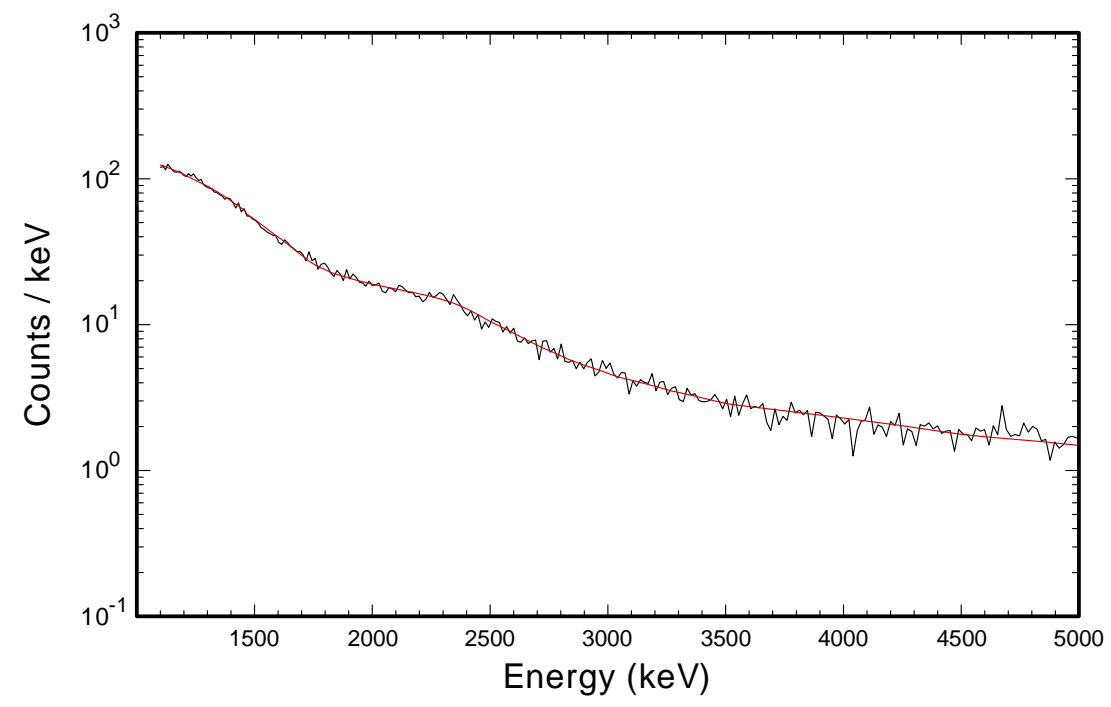

Fig. 3. A background spectrum measured by MCA1 (black) is compared with the fit of the spectrum (red) that was obtained during the energy-calibration procedure.

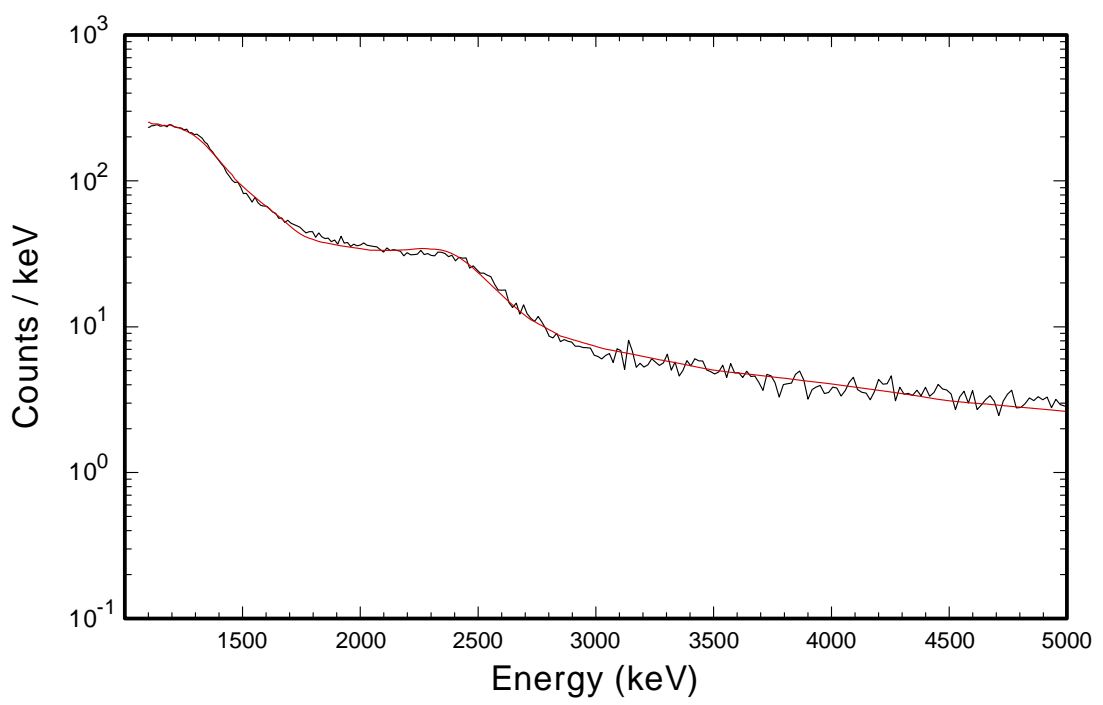

Fig. 4. A background spectrum for the coincident-sum spectrum L1 (black) is compared with the fit of the spectrum (red) that was obtained during the energy-calibration procedure.

The gain and resolution parameters are determined every time a background measurement is recorded. These parameters are applied during subsequent analysis of foreground data. The operator is alerted to the need for gain adjustment if gain parameters drift outside of the normal operating range. Although the LED-gain stabilization performed by the voxelSPECs minimizes drift, adjustment of these parameters is still required occasionally. 


\subsection{Isotope Identification}

Spectra recorded by PVT detectors do not provide distinctive photopeaks of the type that are normally used for isotopic analysis. Nevertheless, energy windowing techniques have been applied successfully to enhance the sensitivity to threat materials without producing excessive false alarm rates. ${ }^{2}$ The application of energy-windowing techniques should benefit from the improved resolution and gain stability of the PVT-NG sensor relative to typical PVT portals. We also evaluated the feasibility of performing isotope identification based on spectra that are acquired with the PVT-NG sensor. Even limited success in this effort could enable enhanced performance. Evaluation of the isotope identification capabilities is based on the performance of the DHSIsotopeID algorithm, which identifies isotopes by fitting spectra with computed spectral templates for one or more isotopes with arbitrary shielding. This analysis algorithm applies a full-spectral analysis technique that utilizes all spectral features without relying exclusively on photopeaks.

\section{Results}

\subsection{Detector Response for Gamma-Ray Sources}

Parameters that are used by the GADRAS gamma-ray detector response function were characterized by recording spectra for a series of calibration sources that were placed 2 meters from the center of the PVTNG sensor. The height was $120 \mathrm{~cm}$ above the ground, which had an asphalt surface. Exact dimensions were applied for the detector dimensions, but parameters that characterize scattered radiation were determined empirically. The detector resolution and peak shape parameters were also characterized to match the measured spectra.

The variable response function parameters were determined by performing a nonlinear regression fit that simultaneously minimized differences between measured and computed spectra for all of the calibration sources. The plots shown in Fig. 5 demonstrate that good agreement between measured coincidencesum spectra and computed spectra was obtained for all of the sources. The resolution was determined to be $18.5 \%$ at $661 \mathrm{keV}$, and the resolution varied as the square-root of energy. The tail that extends to about $2 \mathrm{MeV}$ in the ${ }^{60} \mathrm{Co}$ measurement is associated with coincident detection of the 1173-keV and 1332keV gamma rays that are emitted by ${ }^{60} \mathrm{Co}$. Detection of coincident gamma rays from the same decay chain is not normally observable at a distance of 2 meters, but this effect is measurable in this case because the large PVT detectors represent a substantial solid angle even at a distance of 2 meters. 

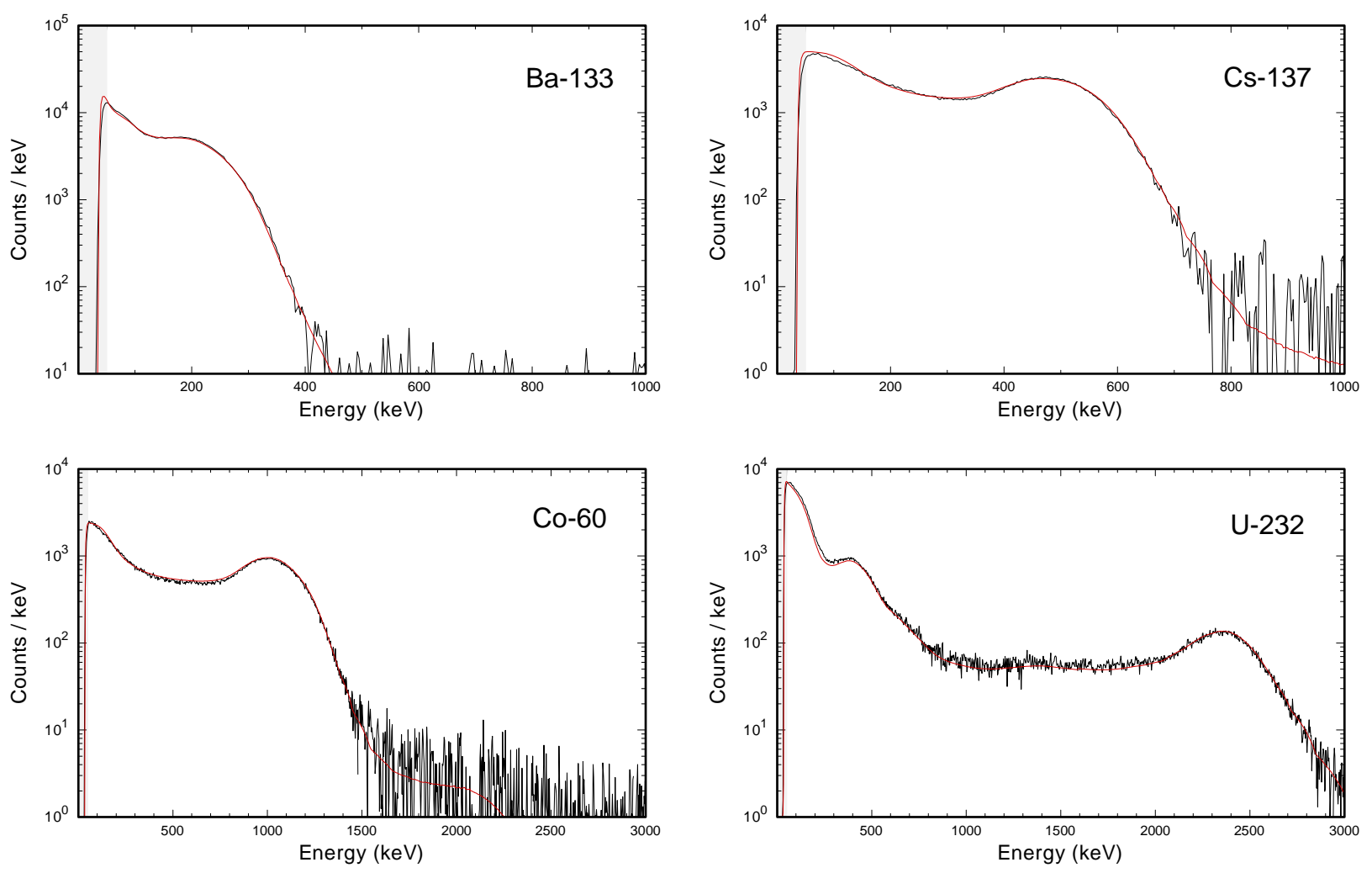

Fig. 5. Comparison of measured background-subtracted spectra (black) with computed spectra (red) for four calibration sources at a distance of 2 meters.

In addition to measurements of the nominal $10 \mu \mathrm{Ci}$ source set for which spectra are displayed in Fig. 5, calibration sources with nominal activities of $100 \mu \mathrm{Ci}$ were also measured to characterize the performance at high count rates. One factor that was determined from these measurements is that the dead time for the coincidence-sum spectra is underestimated by about a factor of two. Although it is only an approximate solution, post-processing was performed so that live times were adjusted to always double the estimated dead time associated with coincidence-sum spectra. Dead times for coincidencesum spectra are greater than dead times for individual MCAs because events are only scored as coincident if the light pulse is processed by MCAs on both ends of the detector.

Another factor that was explored by measurements of the stronger calibration sources is characterization of random pulse pileup effects. GADRAS utilizes a shaping time constant as a basis for performing random pileup calculations. The shaping time parameter only pertains directly to the performance of analogue electronics whereas voxelSPEC MCAs perform digital processing. Nevertheless, the pileup algorithm in GADRAS provides a reasonable estimate for the way random pileup alters the spectrum shape. A shaping time constant of $0.1 \mu \mathrm{s}$ gave the best fit for the calibration spectra. This evaluation implies that random pileup effects can be neglected when the count rate is less than $100,000 \mathrm{cps}$.

\subsection{Response to Neutron Sources}

Fission sources like ${ }^{252} \mathrm{Cf}$ and plutonium emit gamma rays as well as neutrons. The fission gamma-rays are distributed in a continuum that extends to about $9 \mathrm{MeV}$ but decreases exponentially with increasing energy. The intensity of neutrons that are emitted by fission sources peaks at about $1.5 \mathrm{MeV}$. The PVTNG sensor detects neutrons indirectly by measuring spectra for gamma rays that are produced when the neutrons interact within the detector and in surrounding materials. Figure 6 displays spectra that were recorded when the PVT-NG detector was exposed to a bare ${ }^{252} \mathrm{Cf}$ and ${ }^{252} \mathrm{Cf}$ inside $8 \mathrm{~cm}$ of polyethylene $(\mathrm{PE})$. The colored regions in these plots represent the following components: 
Green: measured background spectrum

Red: additional counts that are produced by gamma rays emitted by the radiation sources

Black: additional counts derived from neutron interactions in the detector

The majority of gamma rays above $3000 \mathrm{keV}$ are emitted when neutrons are captured by chlorine in the PVC. The Compton edge at $2 \mathrm{MeV}$ derives from 2223-keV gamma rays that are emitted when neutrons are captured by hydrogen. Hydrogen is present in the PVT detector material itself as well as in the surrounding PVC. Surrounding the ${ }^{252} \mathrm{Cf}$ with $8 \mathrm{~cm}$ of PE reduces the flux of neutrons that strike the detector, as evidenced by reduction of the high-energy gamma ray continuum. The Compton edge at 2 $\mathrm{MeV}$ is more prominent for the moderated ${ }^{252} \mathrm{Cf}$ source because $2223-\mathrm{keV}$ gamma rays are emitted following neutron absorption by hydrogen in the PE that surrounds the ${ }^{252} \mathrm{Cf}$. The count rate above $9 \mathrm{MeV}$ is approximately the same as background in both measurements.
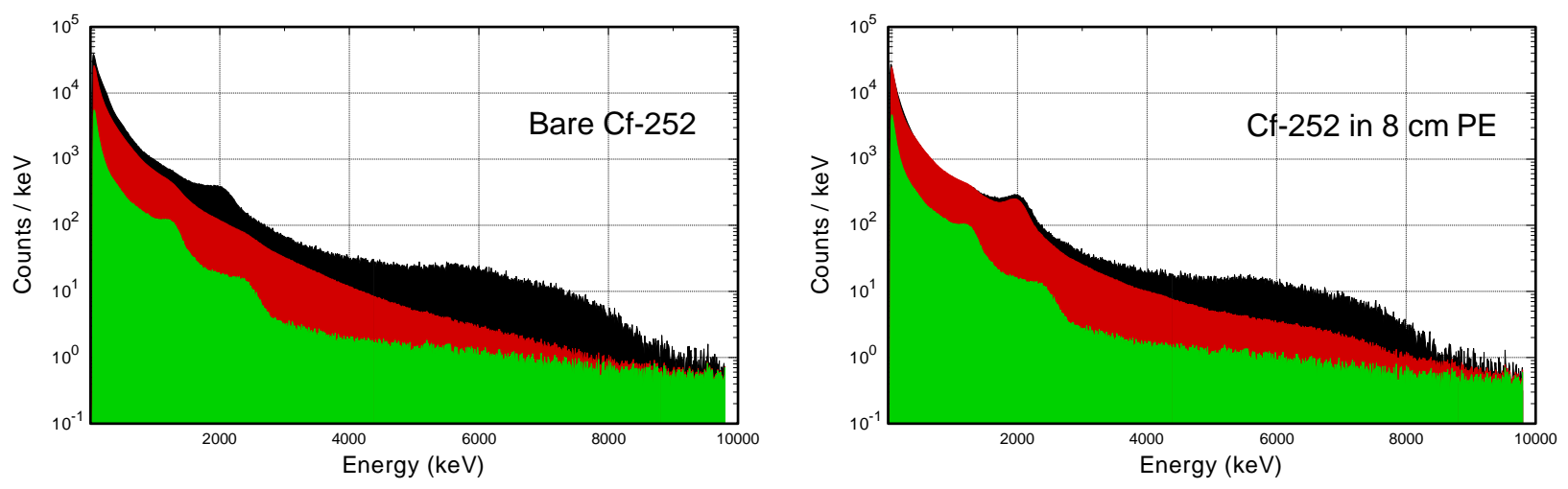

Fig. 6. Comparison of measured spectra (black) with components associated with the computed gamma-ray leakage from the source (red) and the measured background spectrum (green). The detector was exposed to the radiation sources at a distance of 2 meters.

As shown in Fig. 6, neutron sources produce gamma rays at low energy as well as high energy. However, the background gamma-ray count rate is substantially greater below $3000 \mathrm{keV}$, and it is difficult to discriminate between gamma-rays that are produced by neutron interactions and other sources that emit gamma rays below $3000 \mathrm{keV}$. Therefore, $3000 \mathrm{keV}$ represents a nominal energy threshold for detection of neutron sources. Examination of data for several gamma-ray and neutron sources shows that the optimal neutron sensitivity for the PVT-NG sensor is achieved by summing counts in the energy range $3200 \mathrm{keV}$ to $9000 \mathrm{keV}$. In order to reduce false alarms when neutron sources are not present, spectral analysis is performed to estimate the number of counts associated with the high-energy tails produced by $2614 \mathrm{keV}$ gamma rays emitted by ${ }^{232} \mathrm{Th}$ and ${ }^{232} \mathrm{U}$. Although this is generally a small correction, failure to compensate $2614-\mathrm{keV}$ emission can lead to neutron false alarms when strong ${ }^{232} \mathrm{Th}$ or ${ }^{232} \mathrm{U}$ are present. The gross neutron counts that are ascribed to the PVT-NG detector are equal to the number of counts between $3200 \mathrm{keV}$ and $9000 \mathrm{keV}$ in the coincidence-sum spectrum minus the contribution from the 2614-keV gamma rays. The net counts are equal to the gross counts minus the counts in the background spectrum over the same energy range.

GADRAS contains a neutron detector response function that is based on a series of Monte Carlo calculations for an array of ${ }^{3} \mathrm{He}$ tubes with varying thicknesses of PE in front and behind the ${ }^{3} \mathrm{He}$ tubes. The parameters that characterize a neutron detector are the detector area, thicknesses of PE in front and behind the detector, and a scalar efficiency enhancement parameter. The parameters in the response model are adjusted by fitting the number of counts recorded by bare ${ }^{252} \mathrm{Cf}$ and ${ }^{252} \mathrm{Cf}$ inside a series of spherical moderators, which produce a range of neutron energy profiles. The physics associated with neutron detection by the PVT-NG sensor clearly differs from ${ }^{3} \mathrm{He}$ detectors, yet the response function model did a good job of fitting count rates for a series of neutron calibration sources ranging from bare ${ }^{252} \mathrm{Cf}$ to ${ }^{252} \mathrm{Cf}$ inside $8 \mathrm{~cm}$ or PE. Therefore, the performance of the PVT-NG can be compared directly with the performance of other detectors that were characterized previously. 
Figure 7 shows the efficiency of the PVT-NG as a function of neutron energy. The area that is ascribed to a ${ }^{3} \mathrm{He}$-tube neutron detector is normally based on the cross sectional area of the moderator. The moderator that surrounds the ${ }^{3} \mathrm{He}$ tube serves as a converter, which reduces the energy of incident neutrons so that they are absorbed efficiently by ${ }^{3} \mathrm{He}$. In a similar way, the PVT-NG sensor uses the PVC as a converter; in this case, the PVC converts neutrons to high-energy gamma rays that are detected by the PVT. Therefore, the equivalent detector area for the PVT-NG sensor is the cross sectional area of the PVC. The efficiency curves are based on the distances from the calibration source to the detector enclosures rather than the faces of the moderators. The cross sectional area and estimated background count rate that the PVT-NG sensor recorded at the Technical Evaluation Assessment Monitor Site (TEAMS) on Kirtland AFB are listed in Table 1.

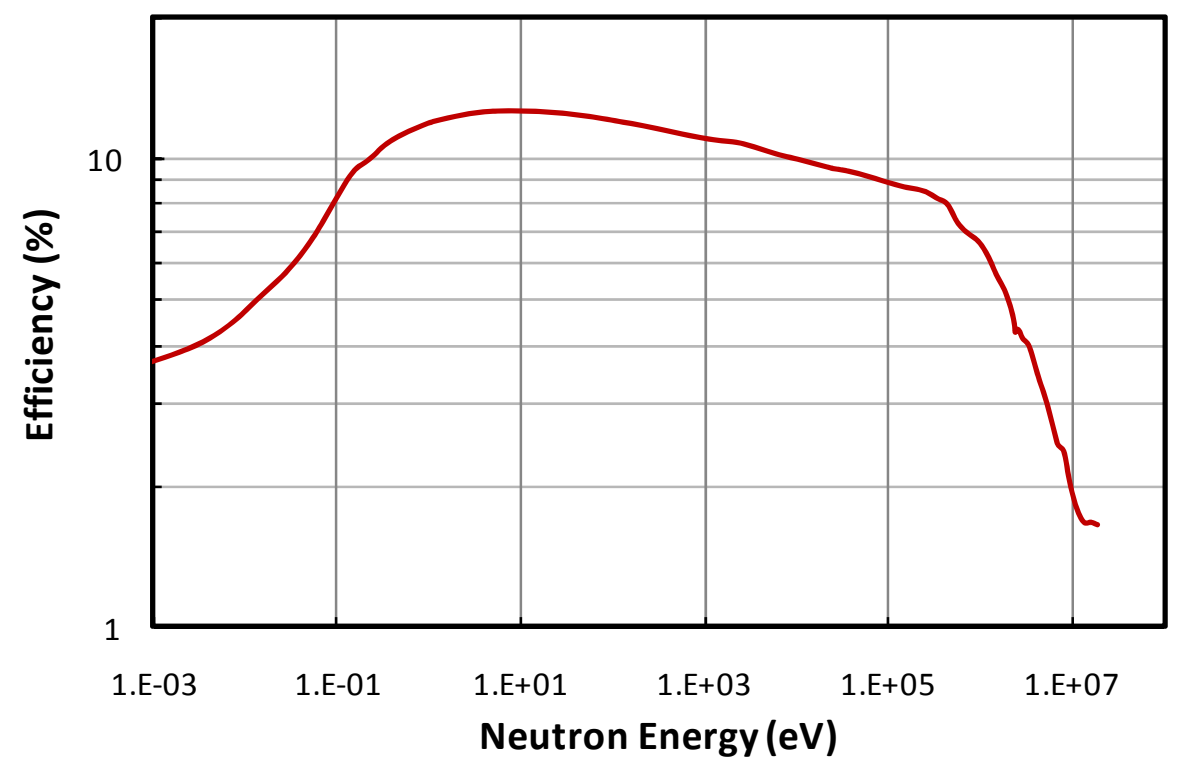

Fig. 7. Estimated neutron detection efficiencies as a function of energy for the PVT-NG sensor. The efficiency represents the percent of neutrons that produce a count relative to the total number of neutrons that strike the face.

Table 1. Cross-sectional area and background neutron count rate for the PVT-NG sensor.

\begin{tabular}{|c|c|c|}
\hline Detector & $\begin{array}{c}\text { Cross-Sectional Area } \\
\left(\mathbf{c m}^{\mathbf{2}}\right)\end{array}$ & $\begin{array}{c}\text { Background Count Rate } \\
\text { at TEAMS } \\
\text { (cps) }\end{array}$ \\
\hline PVT-NG & 7864 & 49 \\
\hline
\end{tabular}

\subsubsection{Sensitivity Evaluation Based on Gross Count Rates}

The neutron detector efficiency curve for the PVT-NG sensor is derived from counts that are recorded in the 3200 to $9000 \mathrm{keV}$ energy range. Although this assessment includes the contribution from fission gamma emitted by the ${ }^{252} \mathrm{Cf}$, Fig. 6 shows that fission gamma rays represent a relatively small component above $3200 \mathrm{keV}$. An advantage of the PVT-NG sensor relative to dedicated neutron detectors is that it is also sensitive to neutron capture gamma rays that are produced in cargo and gamma rays that are emitted following neutron capture by iron in vehicles. However, effects associated with neutron capture in 
cargo and vehicles are difficult to quantify, so the comparative evaluation that is presented in this section is based exclusively on ${ }^{252} \mathrm{Cf}$ inside PE moderators of varying thicknesses.

The sensitivity for neutron detection can be expressed in terms of sigma-deviation units. The sigma value is equal to the number of net counts divided by the uncertainty in the background counts during the measurement interval. Accordingly, the sigma value is given by Eq. (1):

$$
\sigma=\frac{t A}{\sqrt{B t}} \int \varepsilon F d E
$$

where $t$ is the measurement time, $A$ is the cross-sectional area, and $B$ is the background count rate. The parameters $F$ and $\varepsilon$, which represent the radiation flux and the detector efficiency, respectively, are both energy dependent. The radiation flux is expressed in units of neutrons $/ \mathrm{cm}^{2} / \mathrm{s}$. Equation (1) can be rearranged to determine the minimum detectable quantity of ${ }^{252} \mathrm{Cf}\left(Q_{\min }\right)$, as shown below:

$$
Q_{\min }=\frac{4 \pi d^{2} \sigma_{\min }}{\alpha \bar{\varepsilon} A} \sqrt{\frac{B}{t}}
$$

The parameter $d$ is the distance between the source and the face of the detector, $\sigma_{\min }$ is the value of sigma at the detection threshold, and $\alpha$ converts ${ }^{252} \mathrm{Cf}$ activity to neutron emission rate (4320 neutrons $/ \mathrm{s} / \mu \mathrm{Ci}$ ), and $\bar{\varepsilon}$ is the average efficiency. Background count rates must be defined in order to estimate minimum detectable neutron source strengths. The background count rate in the range 3200 to $9000 \mathrm{keV}$ for the PVT-NG sensor was $49 \mathrm{cps}$ at the DTRA TEAMS site, which is an outdoor test facility on Kirtland AFB in Albuquerque.

Table 2 presents the minimum detectable activities of ${ }^{252} \mathrm{Cf}$ in several moderators. Activities that are reported in Table 2 are based on a 4-second exposure at a distance of 2 meters from the face of the detector. These calculations assume that the detection threshold $\left(\sigma_{\min }\right)$ is 5 sigma.

Table 2. Minimum detectable activities $(\mu \mathrm{Ci})$ of ${ }^{252} \mathrm{Cf}$ in various configurations.

\begin{tabular}{||c|c|}
\hline Source Configuration & Minimum detectable activity for ${ }^{252} \mathrm{Cf}(\mu \mathrm{Ci})$ \\
\hline${ }^{252} \mathrm{Cf}$ bare & 4.9 \\
\hline${ }^{252} \mathrm{Cf}$ in $2 \mathrm{~cm} \mathrm{PE}$ & 4.1 \\
\hline${ }^{252} \mathrm{Cf}$ in $4 \mathrm{~cm} \mathrm{PE}$ & 3.7 \\
\hline${ }^{252} \mathrm{Cf}$ in $8 \mathrm{~cm} \mathrm{PE}$ & 3.6 \\
\hline
\end{tabular}

A metric that is often applied when sensitivities of neutron detectors in radiation portals are evaluated is the count rate per nanogram (cps/ng) for ${ }^{252} \mathrm{Cf}$ at a distance of 2 meters. This metric can be misleading because of differences in background count rates. Nevertheless, when expressed in these units, the sensitivity of the PVT-NG sensor to bare ${ }^{252} \mathrm{Cf}$ at a distance of 2 meters is $1.9 \mathrm{cps} / \mathrm{ng}$ according to Eq. (3). The sensitivity for the PVT-NG sensor is $2.3 \mathrm{cps} / \mathrm{ng}$ for ${ }^{252} \mathrm{Cf}$ inside $2.5 \mathrm{~cm}$ of polyethylene.

$$
S=\frac{\varepsilon A}{4 \pi d^{2}} \times 2316\left(\frac{n / s}{n g}\right)
$$

\subsubsection{Sensitivity Evaluation Based on Spectroscopic Analysis}

The sensitivity evaluation presented in Section 3.2.1 is the standard way that the sensitivity of neutron detectors is normally evaluated. This approach is based on the premise that all detection events are of 
comparable significance, which is true of a detector such as ${ }^{3} \mathrm{He}$, where a discharge greater than a threshold energy has equal probability of being produced by a neutron regardless of the energy of the discharge. However, this does not apply to the PVT-NG detector, where an $8 \mathrm{MeV}$ pulse has a higher probability of being produced by a neutron relative to a pulse at $4 \mathrm{MeV}$ due to differences in the background count rates. Consequently, the optimal sensitivity is achieved by estimating uncertainties derived from spectral analysis rather than the gross number of counts in the high-energy region. Figure 8 shows a fit of the region from $1800 \mathrm{keV}$ to $9000 \mathrm{keV}$, which is used to derive statistical parameters related to neutron detection events. The fit includes templates for ${ }^{226} \mathrm{Ra}$ and ${ }^{232} \mathrm{U}$, which produce a few counts in the high-energy regions that are not related to neutron sources. The components of the spectrum that contribute to the detection of neutron sources are:

- the spectrum derived from neutron interactions in the PVT-NG detector

- the fission continuum

- the spectrum produced by 2223-keV gamma rays derived from neutron capture in hydrogen

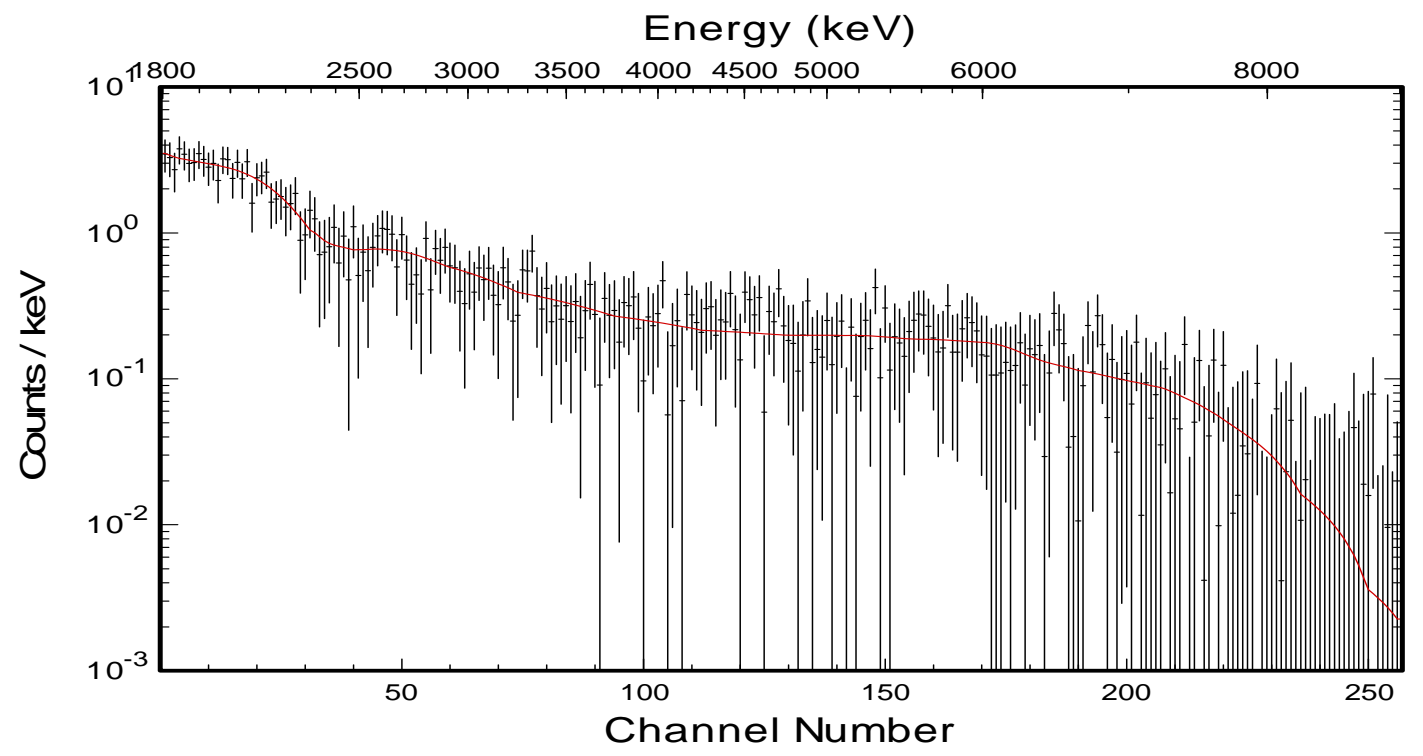

Fig. 8. The red curve shows the fit to the high-energy portion of a spectrum that was measured while a ${ }^{252} \mathrm{Cf}$ source was present and the black error bars represent the stripped spectrum with \pm 1 -sigma standard deviations.

Numerous spectra were analyzed to evaluate neutron detection sensitivities derived from gross count rates and spectral analysis. The spectral analysis method improved the sensitivity by about $10 \%$ relative to the gross count method. The probability for false alarms was similar for the two methods. Therefore, the spectral analysis method was selected as a basis for neutron count rates and uncertainties that are reported by the PVT-NG sensor. The method that is used for estimating the confidence for neutron detection does not affect the sensitivity when expressed in terms of cps/ng, but it does improve the performance with respect to minimum detectable activities of ${ }^{252} \mathrm{Cf}$ by about $10 \%$.

\subsection{Isotope Identification}

The DHSIsotopeID algorithm identifies isotopes by fitting the entire spectrum with computed gamma-ray spectra for various combinations of isotopes with independent shielding. This process does not require the presence of photopeaks, so it is suitable for the analysis of spectra collected with PVT detectors. However, the application had not been tested extensively in association with the analysis of spectra that had been collected with PVT detectors. A few minor changes were made after evaluating spectra that were collected by the PVT-NG sensor. It was not surprising to discover that sources that exhibit very similar emission profiles were difficult to distinguish. Accordingly, many of the uncommon isotopes that are normally contained in the database used by DHSIsotopeID were eliminated to reduce ambiguities. 
This solution would not be ideal if the objective was primarily for isotope identification, but it is suitable for use in a radiation portal, where the objective is to maximize the probability for detecting threat materials while minimizing the probability for false alarms when benign materials are present. Characteristics of the PVT-NG sensor that are beneficial for isotope identification using DHSIsotopelD—namely, improved resolution and gain stabilization-should also be beneficial for application of energy windowing techniques. However, this section of the report focuses on isotope identification capabilities, which have not been explored previously with respect to analysis of spectra that are collected with PVT detectors. A portal that pairs the application of DHSIsotopeID with a PVT detector would alarm on either of two conditions: a) the count rate exceeds a threshold rate; b) the spectral analysis indicates that something besides NORM is present.

Table 3 compares the analysis results with sources that were actually present when measurements were performed. These tests correspond to stationary measurements that were performed when the sensor was exposed to bare calibration sources for varying periods of time at a distance of 3 meters. The isotope identifications were reported with high confidence, as indicated by the string " $(\mathrm{H})$ " following the isotope names. An ambiguity that was encountered with these results is that gamma-ray spectra produced by ${ }^{228} \mathrm{Th}$ and ${ }^{232} \mathrm{U}$ are virtually identical. The DHSIsotopeID algorithm reports the isotope as ${ }^{228} \mathrm{Th}$ if the source was essentially unshielded; otherwise the isotope is called ${ }^{232} \mathrm{U}$. The bare ${ }^{232} \mathrm{U}$ calibration source was identified as ${ }^{228} \mathrm{Th}$ for the 10 and 60-second measurements, which is regarded as a correct identification because it is impossible to distinguish ${ }^{228} \mathrm{Th}$ from ${ }^{232} \mathrm{U}$ under most circumstances regardless of the resolution. The spectrum produced by ${ }^{232} \mathrm{Th}$ is very similar, but it yields additional gamma-rays in the 911-keV region which are not emitted by ${ }^{228} \mathrm{Th}$ and ${ }^{232} \mathrm{U}$. Therefore, it is incorrect to report ${ }^{232} \mathrm{Th}$ when ${ }^{232} \mathrm{U}$ is present, which occurred in the 4-second measurement when all four of the sources were presented simultaneously. Nevertheless, the distinction between ${ }^{232} \mathrm{Th}$ and ${ }^{232} \mathrm{U}$ is also difficult when Nal and HPGe detectors are used in portal applications because ${ }^{232} \mathrm{Th}$ is always present in the background.

Figures 9 through 11 present graphic displays of the analysis results for selected measurements (see the "Figure Number" column of Table 3 ). The measurements are represented by \pm 1 -sigma error bars in each of these plots and colored regions identify components of the spectra that were derived from the analysis. Only a limited number of sources were available for testing, and the analysis results are expected to be less definitive for shielded sources. Nevertheless, it is notable that all of the analysis results were essentially correct in every case (aside from the aforementioned ambiguity associated with ${ }^{228} \mathrm{Th},{ }^{232} \mathrm{Th}$ and ${ }^{232} \mathrm{U}$ ), and the sensitivity of the PVT detector equals or exceeds the performance for some spectroscopic portals that use Nal and HPGe detectors. The weakest of the calibration sources was ${ }^{60} \mathrm{Co}$, which had an activity of $6.6 \mu \mathrm{Ci}$, and the measurements were performed at a distance of 3 meters; these measurements are equivalent to detection of a $3 \mu \mathrm{Ci}$ source at a distance of 2 meters. The large size of the PVT detector largely compensates for its poor resolution relative to $\mathrm{Nal}$ and HPGe-based portals. 
Table 3. Summary of analysis results for measurements for gamma-ray emitting sources.

\begin{tabular}{|c|c|c|c|}
\hline $\begin{array}{l}\text { Measurement } \\
\text { Duration (s) }\end{array}$ & *Source & Isotope Identification & $\begin{array}{c}\text { Figure } \\
\text { Number }\end{array}$ \\
\hline 60 & none & none & \\
\hline 60 & ${ }^{133} \mathrm{Ba}(15.8 \mu \mathrm{Ci} @ 3 \mathrm{~m})$ & Ba133(H) & \\
\hline 60 & ${ }^{137} \mathrm{Cs}(17.8 \mu \mathrm{Ci} @ 3 \mathrm{~m})$ & Cs137(H) & \\
\hline 60 & ${ }^{60} \mathrm{Co}(6.6 \mu \mathrm{Ci} @ 3 \mathrm{~m})$ & $\mathrm{Co60}(\mathrm{H})$ & \\
\hline 60 & ${ }^{232} \mathrm{U}(9.5 \mu \mathrm{Ci} @ 3 \mathrm{~m})$ & Th228(H) & \\
\hline 60 & ${ }^{133} \mathrm{Ba}+{ }^{137} \mathrm{Cs}$ & Cs137(H)+Ba133(H) & \\
\hline 60 & ${ }^{133} \mathrm{Ba}+{ }^{137} \mathrm{Cs}+{ }^{60} \mathrm{Co}+{ }^{232} \mathrm{U}$ & Co60(H)+Th232(H)+Cs137(H)+Ba133(H) & 9 \\
\hline 10 & none & none & \\
\hline 10 & ${ }^{133} \mathrm{Ba}(15.8 \mu \mathrm{Ci} @ 3 \mathrm{~m})$ & Ba133(H) & \\
\hline 10 & ${ }^{137} \mathrm{Cs}(17.8 \mu \mathrm{Ci} @ 3 \mathrm{~m})$ & Cs137(H) & \\
\hline 10 & ${ }^{60} \mathrm{Co}(6.6 \mu \mathrm{Ci} @ 3 \mathrm{~m})$ & $\mathrm{Co60}(\mathrm{H})$ & \\
\hline 10 & ${ }^{232} \mathrm{U}(9.5 \mu \mathrm{Ci} @ 3 \mathrm{~m})$ & Th228(H) & \\
\hline 10 & ${ }^{133} \mathrm{Ba}+{ }^{137} \mathrm{Cs}$ & Cs137(H)+Ba133(H) & \\
\hline 10 & ${ }^{133} \mathrm{Ba}+{ }^{137} \mathrm{Cs}+{ }^{60} \mathrm{Co}+{ }^{232} \mathrm{U}$ & Co60(H)+Th232(H)+Cs137(H)+Ba133(H) & \\
\hline 4 & none & none & \\
\hline 4 & ${ }^{133} \mathrm{Ba}(15.8 \mu \mathrm{Ci} @ 3$ m) & $\operatorname{Ba133}(\mathrm{H})$ & \\
\hline 4 & ${ }^{137} \mathrm{Cs}(17.8 \mu \mathrm{Ci} @ 3 \mathrm{~m})$ & Cs137(H) & \\
\hline 4 & ${ }^{60} \mathrm{Co}(6.6 \mu \mathrm{Ci} @ 3 \mathrm{~m})$ & $\mathrm{Co60}(\mathrm{H})$ & \\
\hline 4 & ${ }^{232} \mathrm{U}(9.5 \mu \mathrm{Ci} @ 3 \mathrm{~m})$ & Th232(H) & \\
\hline 4 & ${ }^{133} \mathrm{Ba}+{ }^{137} \mathrm{Cs}$ & Cs137(H)+Ba133(H) & 10 \\
\hline 4 & ${ }^{133} \mathrm{Ba}+{ }^{137} \mathrm{Cs}+{ }^{60} \mathrm{Co}+{ }^{232} \mathrm{U}$ & Co60(H)+Th232(H)+Cs137(H)+Ba133(H) & 11 \\
\hline
\end{tabular}

*The same sources and distances (3 meters) were used for all measurements. 


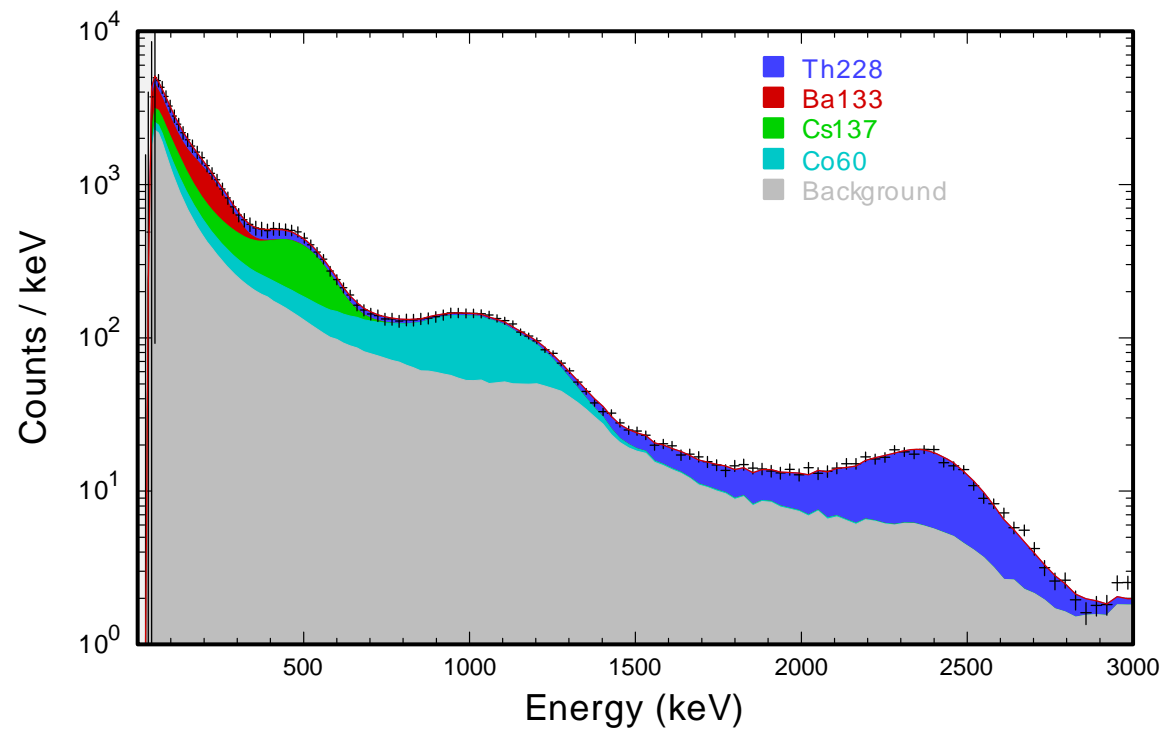

Fig. 9. Comparison of the measured 60 -second spectrum for ${ }^{133} \mathrm{Ba}+{ }^{137} \mathrm{Cs}+{ }^{60} \mathrm{Co}+{ }^{232} \mathrm{U}$ with components of the spectrum derived from analysis with DHSIsotopeID.

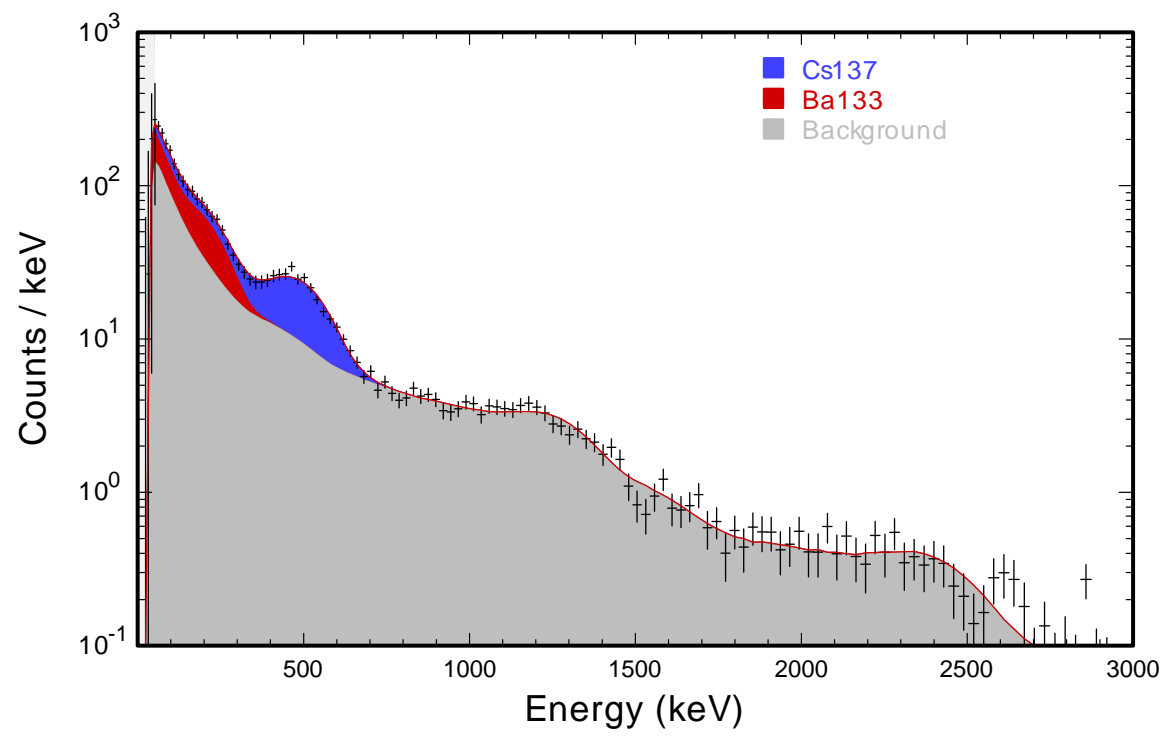

Fig. 10. Comparison of the measured 4-second spectrum for ${ }^{133} \mathrm{Ba}+{ }^{137} \mathrm{Cs}$ with components of the spectrum derived from analysis with DHSIsotopeID. 


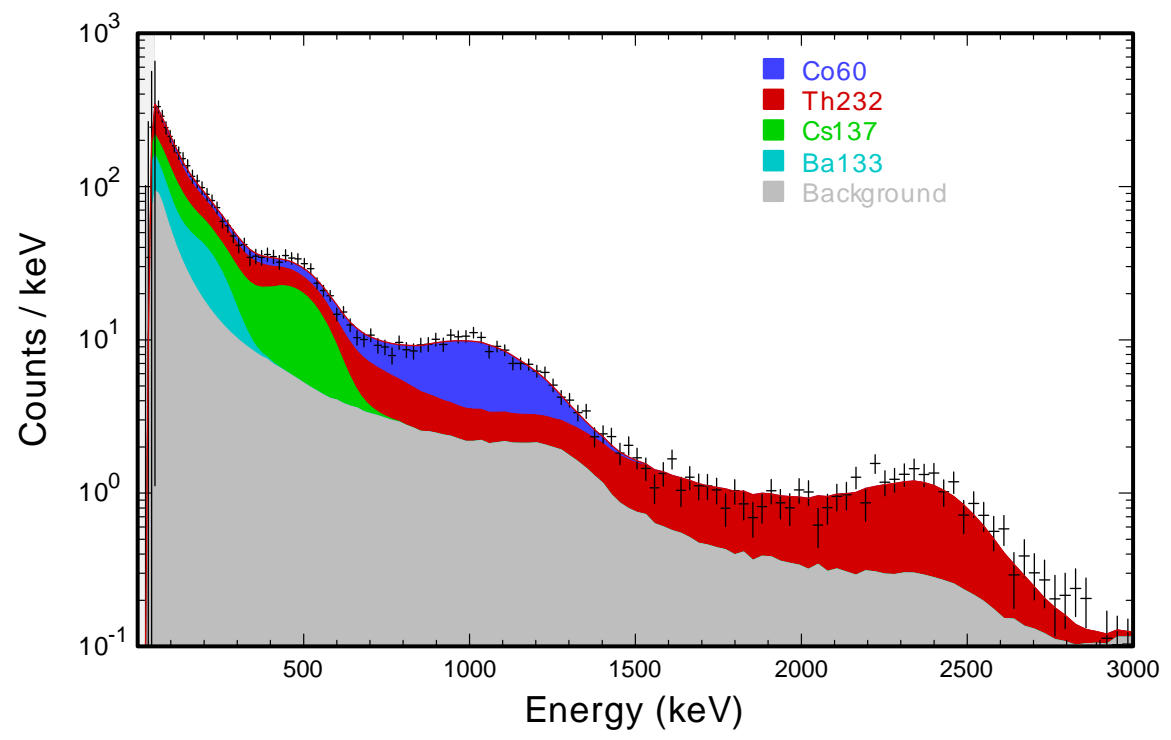

Fig. 11. Comparison of the measured 4-second spectrum for ${ }^{133} \mathrm{Ba}+{ }^{137} \mathrm{Cs}+{ }^{60} \mathrm{Co}+{ }^{232} \mathrm{U}$ with components of the spectrum derived from analysis with DHSIsotopeID.

\subsection{Gain Stability}

Several measurement series were collected to evaluate the performance of the gain stabilization. Despite the use of coolers that are regulated to $27 \mathrm{C}$, substantial gain drift occurred when the gain stabilizers were turned off. This drift is illustrated in Fig. 12, which shows the full scale energy for the two logical detectors following a collection that started at 4 PM on a day when the daytime temperature reached $33 \mathrm{C}$ and the nighttime low was $16 \mathrm{C}$. The stabilizers were turned off during this measurement. Gain drift still occurred, but the magnitude of the drift was reduced by about a factor of two when the gain stabilizers were on.

It was concluded that the variations were probably associated with non-uniform cooling within the NEMA enclosure and intermittent operation of the piezoelectric coolers. Although the coolers might not even be necessary, it seemed advisable to use coolers during the planned mid-summer testing in Nevada. Each piezoelectric cooler is rated for $15 \mathrm{~W}$ of cooling, which is approximately the same as the heat load generated by internal components (about $3 \mathrm{~W}$ per MCA plus heat produced by various power supplies). Therefore, the combined effect of using both coolers only reduces the internal temperature by a small amount relative to the external temperature. There wasn't sufficient time to perform a well-controlled test with temperature probes due to the delivery schedule for independent testing, but we decided that it would be preferable to turn the coolers to their lowest setting of $16 \mathrm{C}$ to minimize the thermal discontinuities that occur while the coolers attempt to regulate the temperature. Figure 13 shows the variation in the full-scale energy during the period 9 AM through $7 \mathrm{AM}$ the following morning. The offset between the full-scale energy of L1 versus L2 is the unintentional result of inaccuracies associated with the algorithm that is used to perform automatic gain adjustments. Both logical detectors exhibit a small systematic variation between day and night, but the magnitude of the variation is only slightly greater than the $50-\mathrm{keV}$ uncertainty associated with the determination of the full-scale energy (estimated to be 50 $\mathrm{keV}$ ). This seems to be the best set of operational conditions, so these are the settings that will be used for the independent evaluation:

- LED-gain stabilizers on, and

- Piezoelectric coolers on with set-points adjusted to16 C 


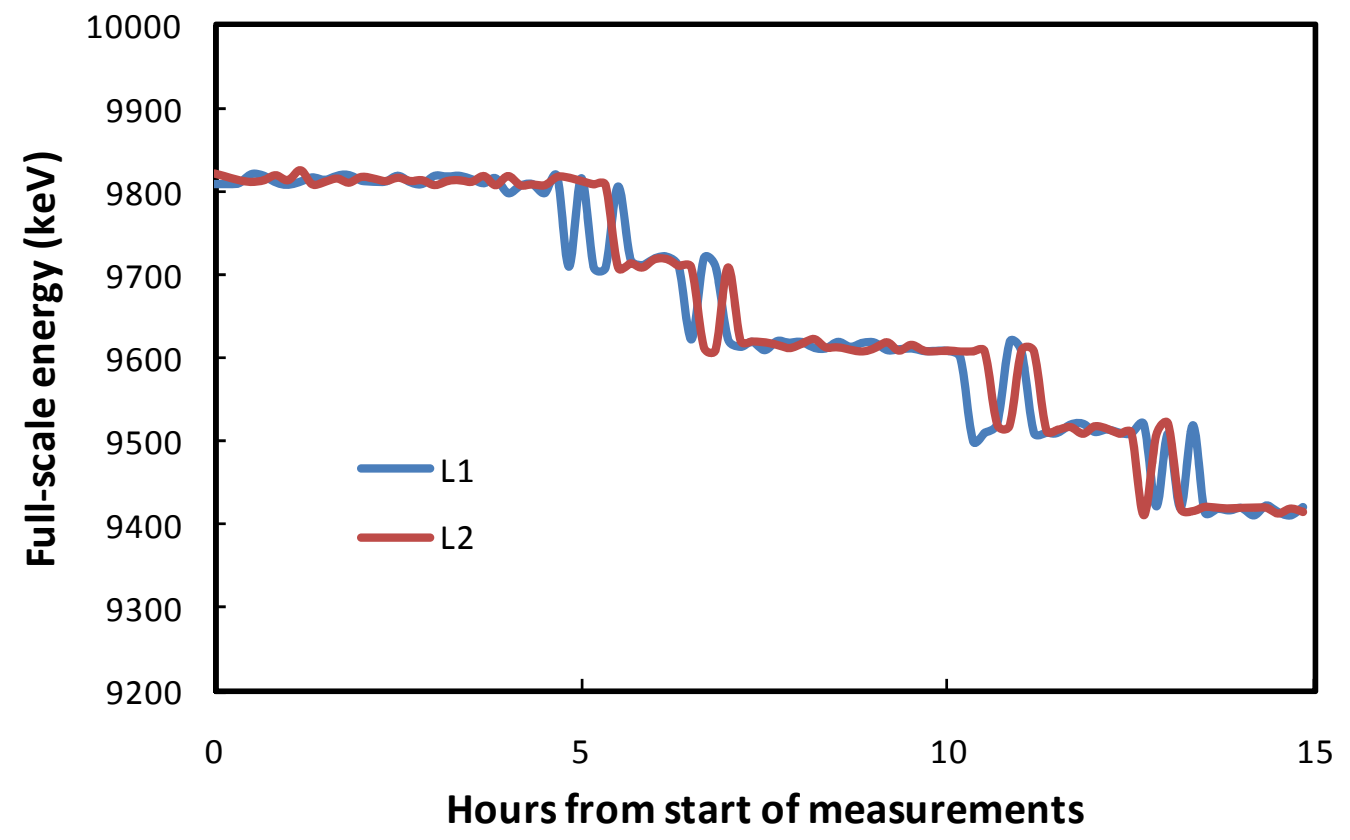

Fig. 12. Full-scale energy for logical detectors L1 and L2 with the LED-gain stabilizer turned OFF and the set-point for the piezoelectric coolers at $27 \mathrm{C}$. The measurement started at 4 PM when the outside temperature was $33 \mathrm{C}$ and terminated at $7 \mathrm{AM}$ the following morning, when the outside temperature was $16 \mathrm{C}$.

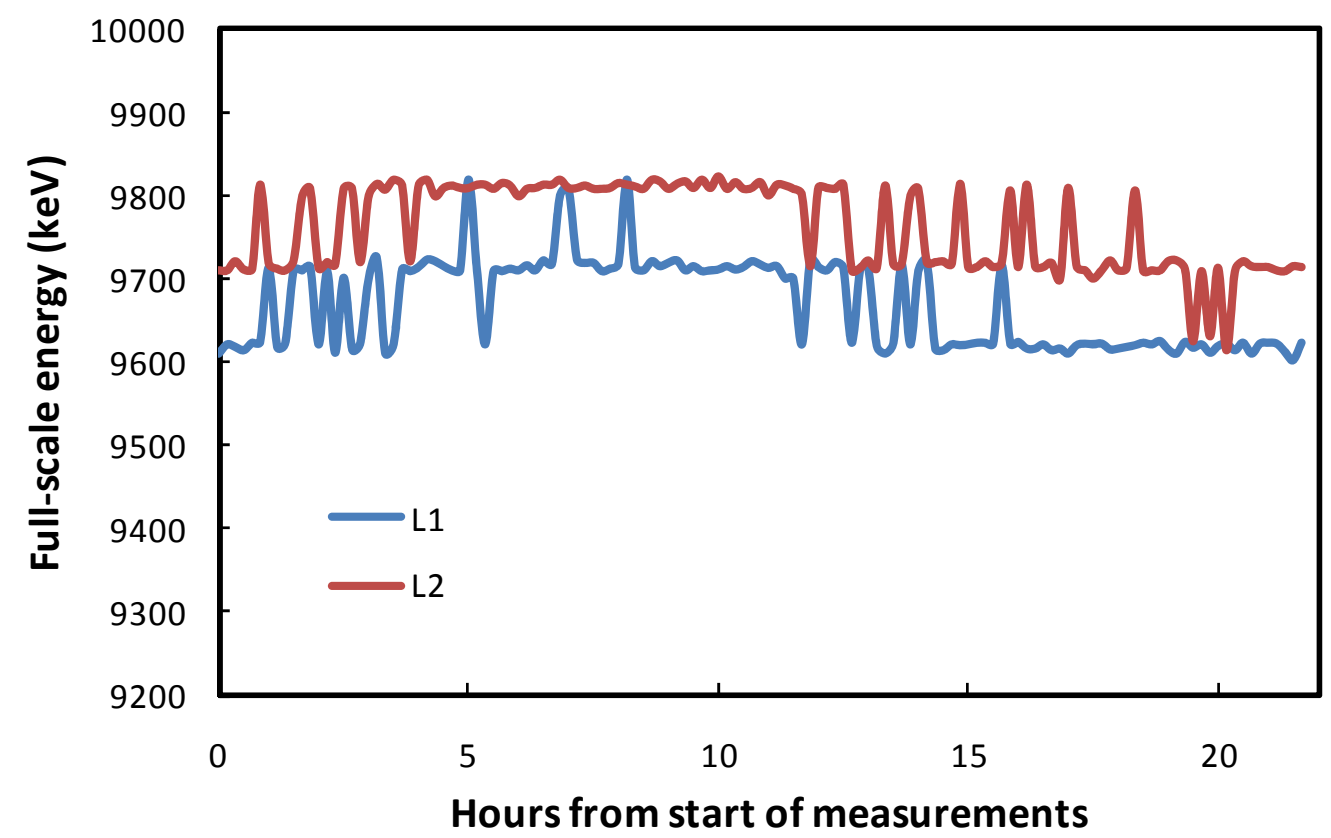

Fig. 13. Full-scale energy for logical detectors L1 and L2 with the LED-gain stabilizer turned ON and the set-point for the piezoelectric coolers at $16 \mathrm{C}$. The measurement started at $9 \mathrm{AM}$ and terminated at $7 \mathrm{AM}$ the following morning. The high for the outside temperature was $33 \mathrm{C}$ and the low was $16 \mathrm{C}$ during this 22-hour measurement sequence. 


\section{Discussion}

\subsection{Neutrons Sensitivity Optimization}

As indicated in Section 3.2, the PVT-NG sensor, as designed and tested in this initial configuration, is about $25 \%$ less sensitive than the DNDO-imposed requirement of $2.5 \mathrm{cps} / \mathrm{ng}$ for bare ${ }^{252} \mathrm{Cf}$ at two meters. Achieving equal performance is not an absolute requirement since ${ }^{3} \mathrm{He}$ is not available for new portals, but obtaining comparable performance is a desirable goal which the authors believe could be achieved. Very little development time was available for the fabrication and testing of the PVT-NG sensor before delivery for an external evaluation. Therefore, it was not possible to make significant changes in response to observations that were made during the performance evaluation. The detector components were also constrained to fit within a 22-cm deep NEMA enclosure, which is an arbitrary restriction because an enclosure with twice this depth could fit within the steel structure of the portal. Results that are presented in this report suggest ways that the neutron detection sensitivity can be improved. Monte Carlo calculations could be used to perform a parametric study of these options.

\subsubsection{Neutron Detection Efficiency}

The detection sensitivity varies directly with the efficiency and inversely with the square-root of the background count rate. Therefore, the greatest payoff can be achieved by improving the efficiency. The results presented in Fig. 7 show that the PVT-NG sensor is less sensitive to high-energy neutrons than the ${ }^{3} \mathrm{He}$-based neutron detectors. An easy way to improve the sensitivity of ${ }^{3} \mathrm{He}$ detectors to high-energy neutrons is to surround the detector with PE moderator. The same principal applies to the PVT-NG sensor because the neutron-capture cross section of chlorine is greatest at low energy, which is also the case for ${ }^{3} \mathrm{He}$. The constraint imposed by the depth of the NEMA enclosure precluded placement of any polyethylene behind the PVT detectors, so the only moderation is provided by hydrogen in the PVT and the 1-inch-thick layer of PVC behind the detector. Neither of these plastics is as good a moderator as PE, which contains 14.4 wt.\% hydrogen compared with 8.4 and 4.8 wt.\% in PVT and PVC, respectively. Enclosing the detector assembly within PE should improve the efficiency; a crude estimate is that the efficiency for un-moderated fission sources can be improved by $50 \%$ for bare ${ }^{252} \mathrm{Cf}$. Insertion of PE behind the NEMA enclosure might also improve the neutron sensitivity without requiring the use of a thicker NEMA enclosure.

The neutron detection sensitivity would be improved by inserting PE or additional PVC in front of the detector, but the additional attenuation would degrade the performance for gamma ray detection. Therefore, alternative approaches must be considered such as inserting steel vanes in front of the detector. Iron in the steel emits high-energy gamma rays much like chlorine, and steel vanes could also reduce the gamma-ray background. Cadmium would be even more effective, but disadvantages associated with using a toxic material like cadmium may be the dominant consideration. Experimentation with $\mathrm{Gd}_{2} \mathrm{O}_{3}$ tile show promise for increasing the neutron cross section while avoiding the use of toxic materials. ${ }^{8}$

Another design option is to adjust the thickness of the PVT detectors. The use of thicker PVT would improve the detection efficiency for high-energy gamma rays. However, hydrogen in the PVT would absorb more of the neutrons before they can be captured by chlorine in the surrounding PVC. Fabricating thicker PVT detectors would also require the use of a special mold since this would not be a common size.

\subsubsection{Background Reduction}

The background for high-energy gamma rays is produced by: naturally occurring neutrons; cosmicinduced gamma rays; and charged particles, which are primarily muons. As part of this development project, an experiment was performed in which 1-inch-thick sheets of borated polyethylene (BPE) were placed outside the steel frame of the portal tower. By stopping some of the background neutrons, the BPE reduces the background associated with neutron-capture reactions in the steel frame and in the PVC. Unfortunately, the count rate for gamma-rays in the energy range $3200 \mathrm{keV}$ to $9000 \mathrm{keV}$ was only reduced by $2 \%$ when the BPE was present. It was concluded that the slight reduction in the count rate 
was not sufficient to justify the additional complexity, so the BPE was not used during any of the other measurements reported in this document.

The current design has a 0.6-cm-thick layer of lead on the back and sides of the detector enclosure. Additional lead shielding would not add substantially to the cost, and the sensitivity for detection of gamma-ray sources would be improved. Additional lead would also attenuate high-energy gamma rays, but interactions with charged particles could have the offsetting effect of producing additional high-energy gamma rays. Therefore, the benefit of additional lead with respect to the neutron sensitivity is not clear, and some experimentation must be performed to optimize the amount of lead shielding.

Muons deposit a gamma-ray energy equivalent of $1.4 \mathrm{MeV} / \mathrm{cm}$ in PVT, so the path length must be less than $6 \mathrm{~cm}$ in order to create a light pulse that is equivalent to a gamma ray below $9 \mathrm{MeV}$. Path lengths as short as this can be derived either from muons with trajectories that pass through the corners of the detectors or muons that are deflected after entering the detector. This component of the background could be reduced by surrounding the PVT assembly with a charged particle detector, and vetoing counts that are in coincidence. However, this approach would add substantial cost and complexity to the sensor, and improve the neutron sensitivity by significantly less than $50 \%$. Given the long penetration range, passive shielding has little effect on muons. Hence, reducing the background component produced by muons is probably impractical.

\subsection{Isotope Identification}

The isotope identification results are very encouraging. Regardless of how the data is processed, the improved resolution, gain stability and gamma-ray detection efficiency are beneficial characteristics. These features may lead to substantial improvement in the sensitivity for detection of threat sources based on gamma-ray measurements. Additional measurements, including measurements of special nuclear materials, shielded sources, and vehicles with normal cargo are required to quantify the extent to which isotope identification could improve the performance relative to existing PVT-based portals.

\subsection{Gain Stability and Resolution}

Data that were collected during this study were used to evaluate the performance of the LED-gain stabilization. Aside from the obvious need to eliminate abrupt gain transitions that were observed occasionally during this evaluation, the method for applying gain stabilization could also be improved from a system perspective. As shown in Section 2.3, coincidence-sum spectra exhibit distinctive spectral features, which enable more precise determination of the gain relative to what can be achieved for the individual MCAs. However, in the current sensor, gains are adjusted by inspecting background spectra that are recorded by the individual MCAs, and errors in the gain adjustments limit the resolution that is achieved for the coincidence-sum spectra. A more effective way to utilize the gain stabilization is to mount a single LED at the center of each of the PVT detectors so that gains for the MCAs on the two ends of the PVT are matched precisely. This would eliminate the need to perform energy calibration for spectra acquired by the individual MCAs, and calibration would only need to be performed for the sumcoincidence spectra.

\subsection{Count-Rate Limitations}

Relative to other PVT portals, the PVT-NG sensor has exceptional performance for detection and identification of gamma-ray emitting sources, and relatively minor changes can increase the neutron detection sensitivity to rival ${ }^{3} \mathrm{He}$-based neutron detectors. However, a fundamental limitation associated with using one type of detector for both gamma-ray and neutron detection is that the performance degrades at high count rates. The dead time for the current system increases rapidly when the count rate approaches $100,000 \mathrm{cps}$. This limitation is imposed by the bottleneck associated with Ethernet traffic, which passes all of the time-tagged data to the EventBuilder application running on a computer that constructs both individual and sum-coincidence spectra. It should be possible to operate the sensor at $1,000,000 \mathrm{cps}$ using electronics that are designed specifically for use in an operational portal rather than a proof-of-principal prototype. Compensation for random pulse pileup would be required at this count rate, but this should not be a severe challenge based on characterization data for the current PVT-NG sensor. However, operation at count rates that are substantially greater than $1,000,000 \mathrm{cps}$ without 
degraded performance will probably not be possible. At a distance of 2 meters, $a{ }^{137} \mathrm{Cs}$ source with an activity of $4 \mathrm{mCi}$ would produce 1,000,000 cps, and the corresponding dose rate at the face of the detector would be $300 \mu \mathrm{R} / \mathrm{hr}$.

The presence of a strong radiation source seriously degrades the ability to detect weak threat sources that may also be present, and a radiation source that produces a dose of $300 \mu \mathrm{R} / \mathrm{hr}$ at a distance of 2 meters represents a threat in itself. Hence, any vehicle emitting radiation with this intensity would be directed to a secondary inspection station. Therefore, the fact that the neutron detection capabilities are degraded in high gamma-ray field has no impact on procedures that would be followed after the detection event at a primary inspection station. Nevertheless, radiation portal performance specifications are currently based on ${ }^{3} \mathrm{He}$ detectors, which can operate in gamma ray fields of $20 \mathrm{mR} / \mathrm{hr}$. The PVT-NG sensor would exhibit almost $100 \%$ dead time in a gamma-ray field of this intensity.

\section{Conclusions}

The limited availability of ${ }^{3} \mathrm{He}$ imposes a pressing need to transition to alternative neutron detection technologies. The PVT-based sensor that is evaluated in this investigation is about $25 \%$ less sensitive to bare ${ }^{252} \mathrm{Cf}$ than the DNDO requirement of $2.5 \mathrm{cps} / \mathrm{ng}$. Neutron sensitivity could be improved by including additional moderator and/or materials that emit gamma rays following neutron capture reactions. Incorporation of additional gamma-ray shielding could also be beneficial. In addition to responding to neutrons, the PVT-NG sensor also records high-energy gamma rays emitted by vehicles and cargo, so the sensitivity to neutron sources could surpass ${ }^{3} \mathrm{He}$-based neutron detectors when neutron sources are transported in vehicles.

Many of the features that enable neutron detection using the PVT detector are also beneficial for gammaray detection. Current portals use energy-windowing algorithms to improve the sensitivity for threat materials without creating an excessive number of false alarms. The performance of algorithms of this type could be improved by utilizing features associated with the PVT-NG sensor, including:

- gain stabilization

- improved resolution

- greater detection efficiency for high-energy gamma rays

This study showed that isotope identification based on analysis of gamma-ray spectra recorded by the PVT-NG is possible, and the large detector area enables the identification of weak sources. Although the sensor is not expected to achieve isotope specificity comparable to Nal and HPGe detectors, spectral analysis shows promise for enabling improved sensitivity to threat materials relative to current PVT-based portals.

The PVT-NG sensor is a proof-of principal system that was developed to demonstrate that PVT can be used for simultaneous gamma-ray and neutron detection. Several changes are suggested that could improve the performance. However, a fundamental limitation associated with the approach of using one type of detector for both gamma-ray and neutron detection is that the ability to identify neutron sources degrades when a strong gamma-ray source is present. This limitation does not influence the concept of operations for primary inspection stations because vehicles containing strong radiation sources would be diverted for secondary inspection regardless of whether a neutron source is present. However, reliable detection of neutron sources cannot be achieved in a $20 \mathrm{mR} / \mathrm{hr}$ gamma-ray field, which is a requirement that derives from the performance of ${ }^{3} \mathrm{He}$ detectors rather than from operational considerations for primary inspection stations. 


\section{Appendix A: Uniformity of Detector Response}

Upon the suggestion of Guntram Pausch of FLIR, two MCAs were used to read spectra from each of the PVT detectors because the coincidence sum spectrum is relatively insensitive to non-uniformities of the light collection efficiency. Therefore, the resolution is improved relative to what would be achieved using only one MCA per detector. The uniformity of the response was investigated by recording spectra while a collimated ${ }^{232} \cup$ source was placed at various locations along the length of one of the PVT detectors. The full-scale energies (FSE) and resolutions for spectra recorded by MCA1, MCA2 and the L1 spectra were determined by fitting the spectra in the range 1 to $5 \mathrm{MeV}$ with computed spectra for ${ }^{232} \mathrm{U}$. Backgrounds were subtracted from the spectra before fitting the data to eliminate the bias that would be imposed by background radiation, which is distributed uniformly across the length of the detector. Figures A1 through A3 compare spectra that were recorded by MCA1 and MCA2 with the coincident sum spectrum, which is designated logical detector 1 (L1). Although the coincidence sum spectra are substantially less sensitive to the location in which gamma rays strike the detector, the spectra still exhibit sensitivity to the position along the detector axis, particularly when the source is close to the end of the detector.

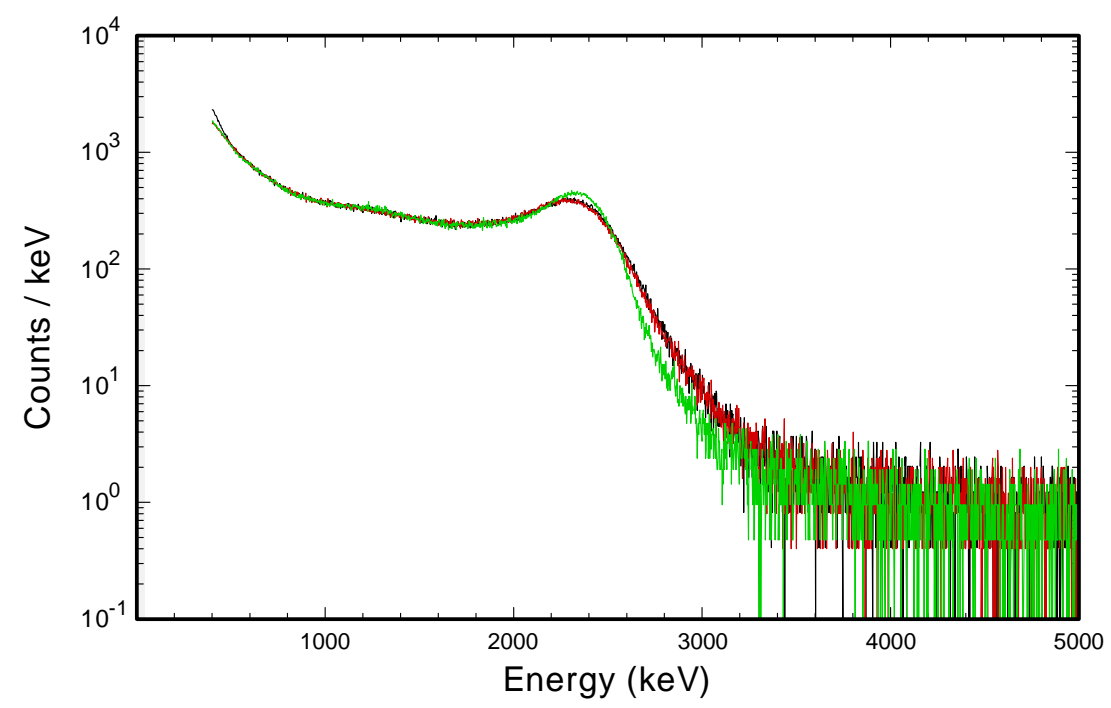

Fig. A1. When a collimated ${ }^{232} \mathrm{U}$ source is placed at the center of one of the PVT detectors, the centroid of the Compton edge from 2614-keV gamma rays is about the same for spectrum L1 (green) and spectra for the individual MCAs. The spectra from the two MCAs are virtually identical in this plot (MCA1 is red and MCA2 is black). 


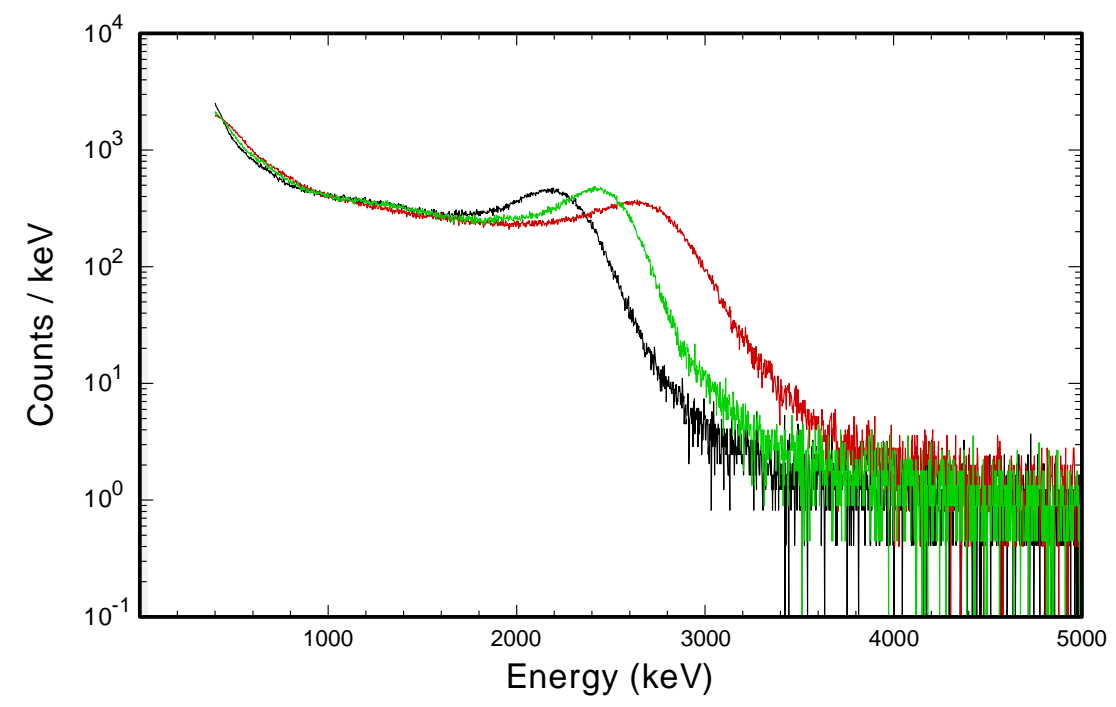

Fig. A2. Spectra that are recorded when the collimated ${ }^{232} U$ source is placed $36 \mathrm{~cm}$ from the end of the PVT detector to which MCA1 is attached: spectrum L1 is green; the spectrum recorded by MCA1 is red; the spectrum recorded by MCA2 is black.

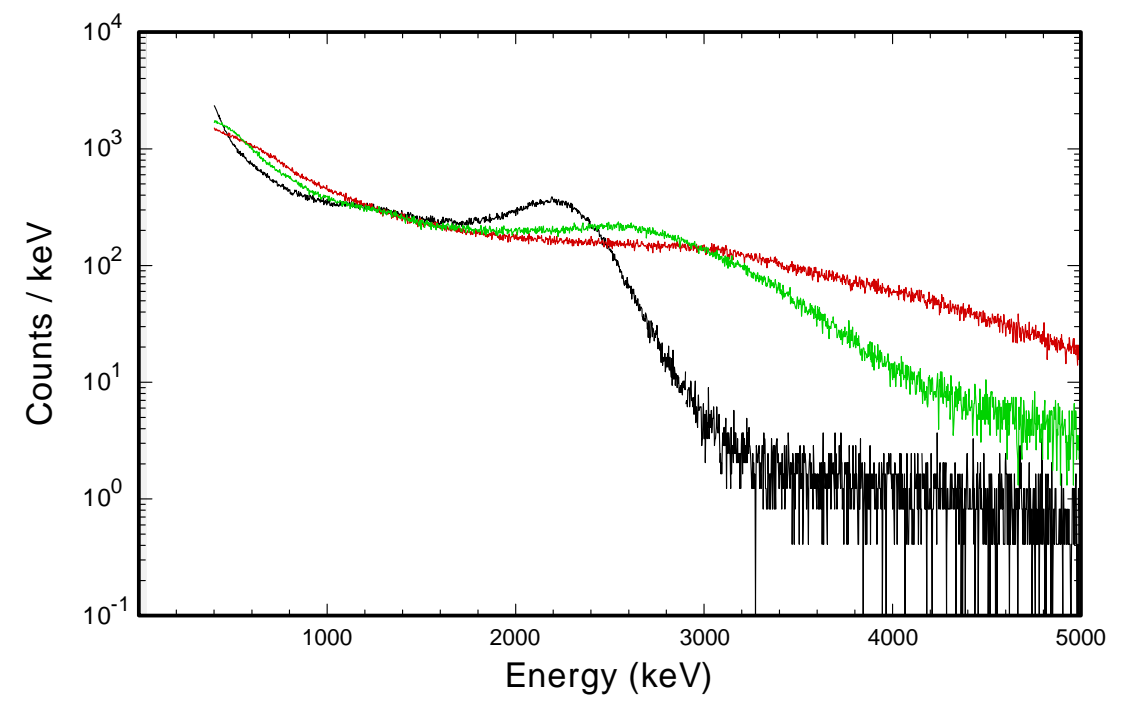

Fig. A3. Spectra that are recorded when the collimated ${ }^{232} \mathrm{U}$ source is placed $5 \mathrm{~cm}$ from the end of the PVT detector to which MCA1 is attached: spectrum L1 is green; the spectrum recorded by MCA1 is red; the spectrum recorded by MCA2 is black. 
Figure A4 shows the dependence of FSE on the source position for MCA1, MCA2 and L1. Gains were adjusted before these measurements were made so that the FSE for the three types of spectra coincide when the source is placed at the center of the detector. Figure A5 shows the full-scale energy for the L1 relative to FSE when the source is placed at the center of the detector as a function of the ratios of FSE for MCA2 relative to MCA1.

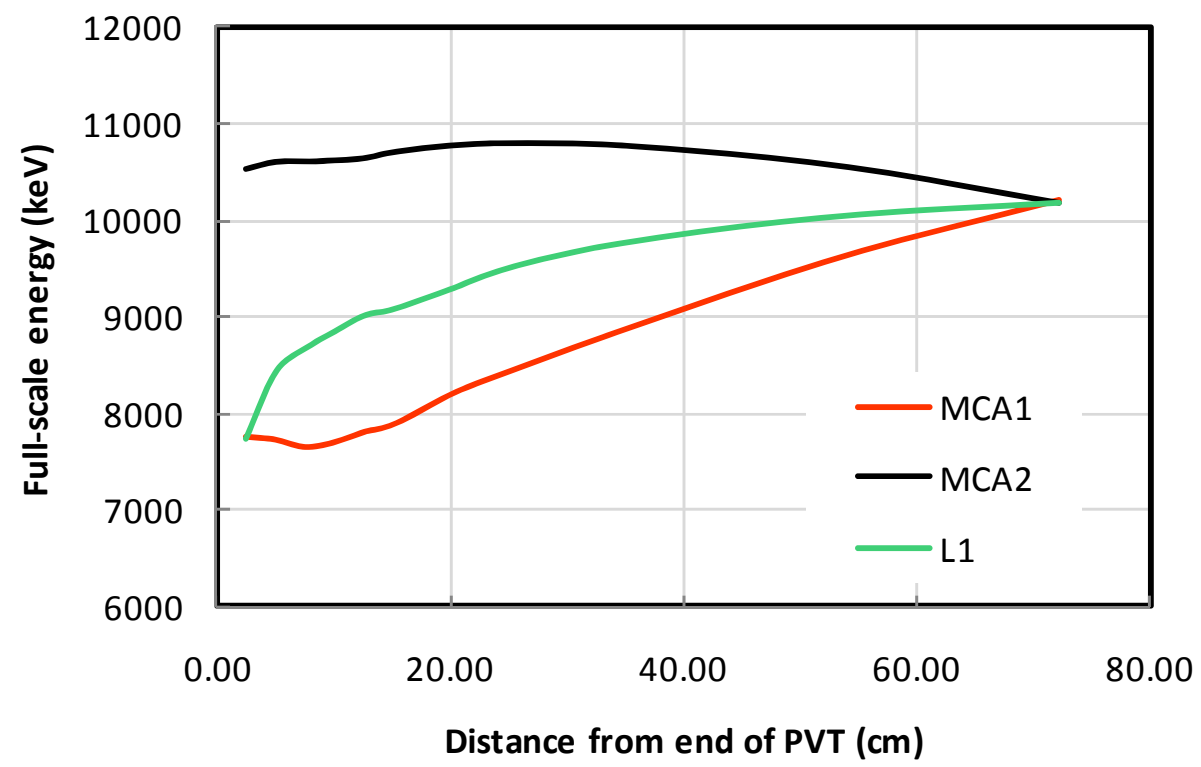

Fig. A4. The full-scale energies for the nearest MCA (MCA1), the furthest MCA (MCA2) and the coincidence sum spectrum L1 are graphed as a function of the distance of the ${ }^{232} \mathrm{U}$ source from the end of the PVT detector.

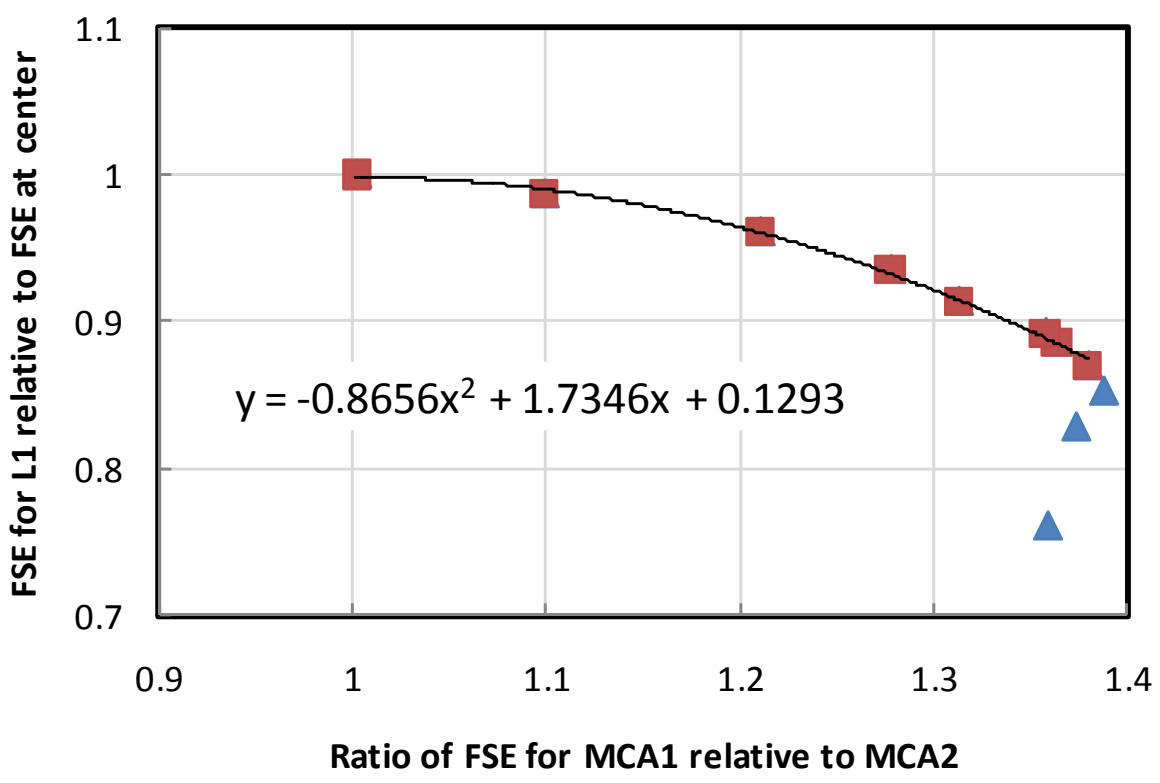

Fig. A5. The relative FSE for L1 is graphed as a function of FSE for MCA1 relative to MCA2. The trend line and the corresponding equation are derived by fitting the data points shown in red. The blue triangles correspond to measurements that were recorded within $8 \mathrm{~cm}$ of the end of the detector. 
Resolutions for each of the three types of spectra that are discussed in this appendix broadened substantially when the ${ }^{232} U$ source was placed within $10 \mathrm{~cm}$ of the end of the PVT detector. Figure A6 graphs resolutions as a function of distance from the end of the PVT. The coincidence sum spectrum, L1, exhibits a resolution of about $18.5 \%$ when the source is placed near the center of the detector and the resolution increases slightly as the source is moved toward the end of the PVT. The projection of ${ }^{232} \mathrm{U}$ measurements to estimate the resolution at $661 \mathrm{keV}$ assumes that the resolution varies as the squareroot of the gamma-ray energy, which was established in Section 3.1.

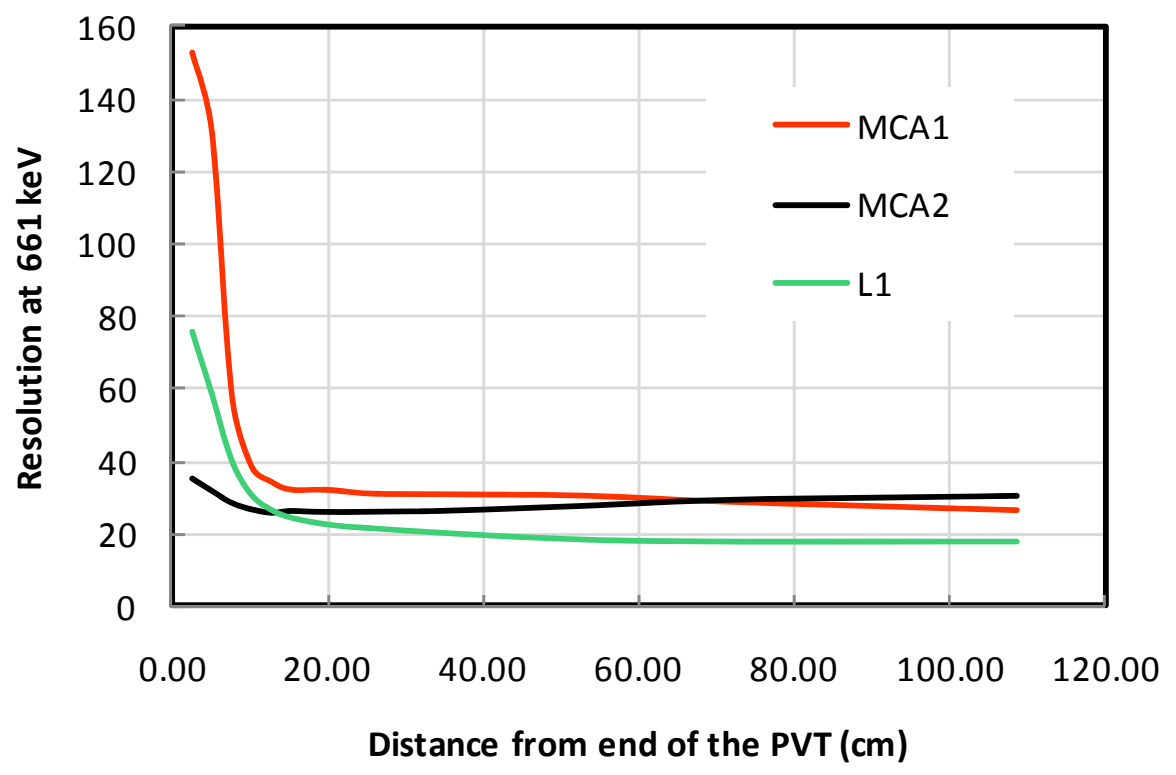

Fig. A6. The resolution for individual MCAs and L1 are presented as a function of the distance of the ${ }^{232} \mathrm{U}$ relative to the end of the PVT.

Observations regarding the location dependence of the detector response suggest ways that the performance of the detector can be improved. Shielding the ends of the detector is one approach to addressing the observation that resolutions degrade when the gamma-rays interact near the end of the detector. Accordingly, 1.3-cm-thick sheets of lead that cover regions extending to $10 \mathrm{~cm}$ from the ends of the detector were added after performing measurements that are discussed in this appendix. In principal, resolutions could also be improved by adjusting the apparent energy of light pulses according to the trend line shown in Fig. A5. The parameter $x$ in the trend line is equal to the following:

$$
x=\operatorname{Max}\left[\frac{E(M C A 1)}{E(M C A 2)}, \frac{E(M C A 2)}{E(M C A 1)}\right]
$$

Accordingly, an improved estimate of the energy that was deposited in the detector is given by deriving the coincident sum energy L1 from the following relationship:

$$
E(L 1)=\left[\frac{E(M C A 1)+E(M C A 2)}{2}\right] \times\left(0.1316+1.734 x-0.8656 x^{2}\right)
$$

The offset term in the trend line represented in Eq. (A2) was adjusted slightly relative to Fig. A5 so that the value of the trend line is exactly equal to 1 when $x$ is equal to 1 .

The trend line in Fig. A5 represents the macroscopic observation based on the sum of numerous light pulses, but this trend should also apply to individual detection events. However, the intensities of signals recorded by MCA1 and MCA2 have associated statistical uncertainties. Adjusting the energy of a light pulse as shown in Eq. (A2) could degrade the resolution if the uncertainties lead to an incorrect 
representation of the light collection efficiency for the individual pulse. The approach of using the ratio of light pulses to adjust the energy for the associated logical detector merits investigation, but this approach is not likely to yield substantial performance improvements.

\section{References}

1. Dean J. Mitchell, Lee T. Harding, and Kenneth R. Smith, "Neutron Detection with Gamma-Ray Spectrometers for Border Security Applications", IEEE Trans. on Nuc. Sci., Vol. 57, No. 4, p. 2215 (August 2010).

2. James Ely, Richard Kouzes, John Schweppe, Edward Sinciliano, Denis Strachan, and Dennis Weier, "The use of energy windowing to discriminate SNM from NORM in radiation portal monitors", Nucl. Inst. and Methods in Phys. Res. A 560, 373-387 (2006).

3. Dean J. Mitchell and John Mattingly, "Rapid Computation of Gamma-ray Spectra for OneDimensional Source Models", American Nuclear Society: 2008 Annual Meeting (June 2008).

4. voxeISPEC is a trademark of FLIR Radiation $\mathrm{GmbH}$.

5. Katja Roemer, G. Pausch, C. Herbach, Y. Kong, R. Lentering, C. Plettner, J. Stein, M. Moszynski, L. Swiderski and T. Szczesniak, "A Technique for Measuring the Energy Resolution of Low-Z Scintillators", IEEE Nuclear Science Symposium (2009).

6. G. Pausch, K. Roemer, C. Herbach, Y. Kong, R. Lentering, J. Stein, "Characterization and Calibration of Large-Volume PVT Detectors Using Tagged Gamma Sources", IEEE Nuclear Science Symposium (2011).

7. Dean J. Mitchell and George P. Lasche, Calculation of Background Radiation for Gamma-Ray and Neutron Detectors, Sandia National Laboratories Report SAND2008-4478 (2008).

8. Guntram Pausch of FLIR Radiation, private communication (June, 2011). 


\section{Distribution (electronic copies)}

1 MS0782 Charles Brusseau 6633

1 MS0782 Dean Mitchell 6633

1 MS0899 Technical Library 9536

cabruss@sandia.gov

djmitch@sandia.gov

External

Greg Slovik

DHS/DNDO Gregory.Slovik@dhs.gov

Hartmut Brands FLIR

Hartmut.Brands@flir.com

Thomas Glaser

FLIR

Thomas.Glaser@flir.com

Guntram Pausch

FLIR

Guntram.Pausch@flir.com

Juergen Stein

FLIR

Juergen.Stein@flir.com

Greg Wortman

FLIR

Greg.Wortman@icxt.com 


\section{Sandia National Laboratories}

